\title{
Gut and airway microbiota and their role in COVID-19 infection and pathogenesis: a scoping review
}

\author{
Tik Fung Dave Liu ${ }^{1}$ Elena Philippou ${ }^{2,3} \cdot$ Ourania Kolokotroni $^{1} \cdot$ Georgios Siakallis ${ }^{1} \cdot$ Kenan Rahima ${ }^{4}$. \\ Constantina Constantinou ${ }^{1}$ (D)
}

Received: 16 July 2021 / Accepted: 9 October 2021 / Published online: 20 October 2021

(c) The Author(s), under exclusive licence to Springer-Verlag GmbH Germany 2021

\begin{abstract}
Background The SARS-CoV-2 virus is responsible for the COVID-19 pandemic. Researchers have been studying the pathogenesis of the virus with the aim to improve our current diagnosis and management strategies. The microbiota have been proposed to play a key role in the pathogenesis of the disease.

Purpose To investigate and report on the current available evidence on any associations between the gut and/or airway microbiota and the pathogenesis of COVID-19.

Methods Using a predefined protocol in compliance with the PRISMA guidelines, a search was conducted on MEDLINE, Science Direct, DOAJ and Cochrane databases on primary research studies assessing the association between COVID-19 infection and the gut and/or airway microbiota.

Results Twenty-two studies were included in the current review; nineteen studies concluded an association between the gut and/or airway dysbiosis and SARS-CoV-2, while 3 studies failed to observe a significant association between the airway microbiome and SARS-CoV-2 infection. Specifically, most studies reported a decrease in microbial diversity and therefore development of intestinal dysbiosis in COVID-19-positive patients compared to healthy controls as well as a possible association between increased intestinal dysbiosis and disease severity.

Conclusion During infection with SARS-CoV-2, there are significant changes in the composition of the gut and airway microbiota. Furthermore, the gut microbiota may have a more important role than the airway microbiota in COVID-19 infection. In the future, studies should be more carefully designed to derive more conclusive evidence on the role of the gut and airway microbiota following infection with SARS-CoV-2 which will lead to the formulation of better management strategies in combating COVID-19.
\end{abstract}

Keywords COVID-19 $\cdot$ SARS-CoV-2 $\cdot$ Gut microbiota $\cdot$ Airway microbiota $\cdot$ Dysbiosis

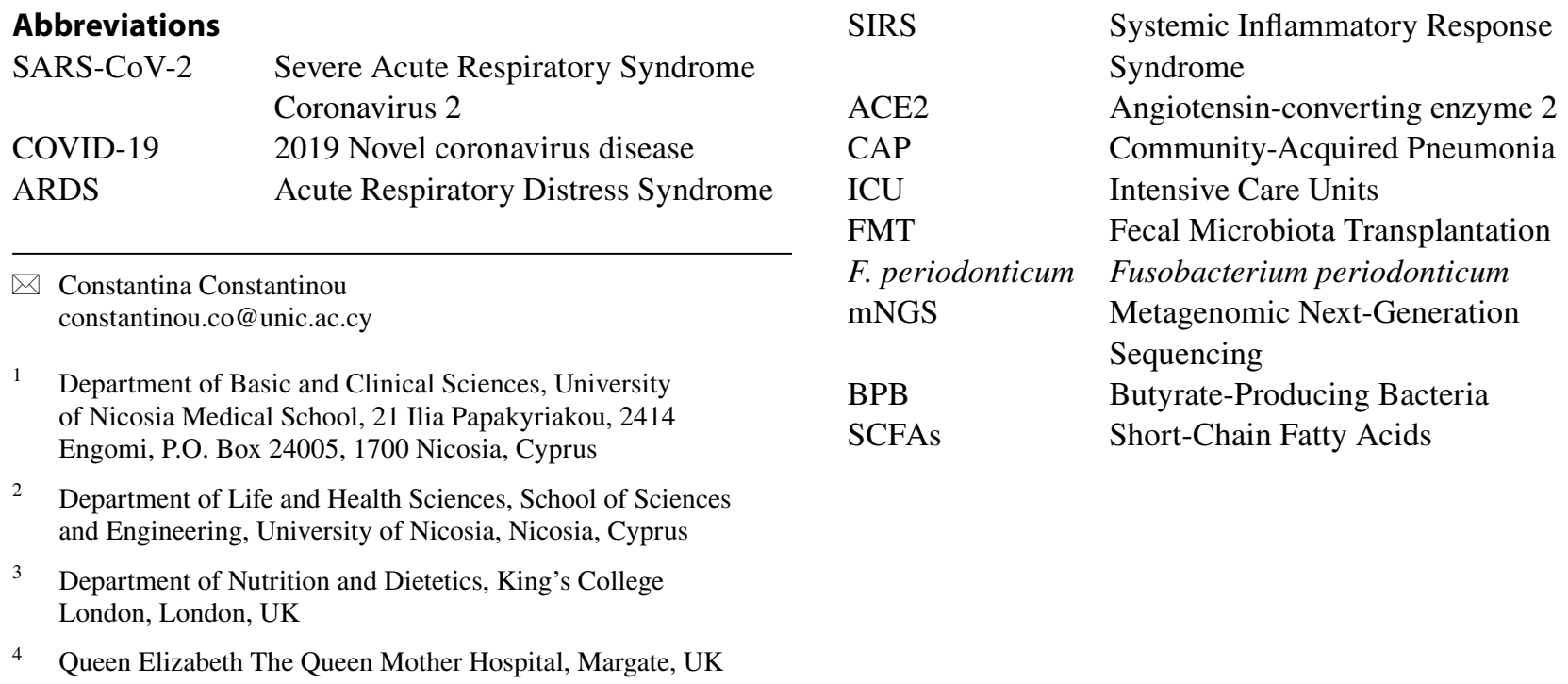




\section{Introduction}

SARS-CoV-2 is a novel beta coronavirus, responsible for the 2019 novel coronavirus disease (COVID-19) [1]. SARS-CoV-2 can cause Acute Respiratory Distress Syndrome (ARDS) which may develop into Systemic Inflammatory Response Syndrome (SIRS), subsequently leading to multi-organ failure [2] and death [3-5]. COVID-19 has had a severe impact on health [6]; from the beginning of the pandemic until October 1, 2021, COVID-19 has affected 220 countries accounting for $233,503,524$ cases and causing 4,777,503 deaths [7].

The respiratory tract is believed to be the main mode of entry of the virus [8]. Angiotensin-Converting Enzyme 2 (ACE2) which is expressed heavily on both the respiratory and the gastrointestinal epithelium is the receptor to which the viral spike binds [9]. Respiratory droplets are believed to be the major source of transmission; yet faecal transmission may also play a role in transmission [10]. Therefore, understanding the association between the gut and airway microbiota and COVID-19 pathogenesis provides an important foundation to formulate better diagnostic and management strategies on combating the pandemic [11].

The microbiota in our body consist of bacteria, archaea, fungi and viruses that can affect the host health and help prevent diseases [12]. The microbiota play a pivotal role in the gut by modulating immune homeostasis, thereby providing an overall protection from pathogens [13, 14]. On the other hand, the airway microbiome is believed to be part of the first barrier against respiratory viral infections and disease progression [15]. In fact, evidence suggests that gut and airway microbiota may also play a role in the pathogenesis of other respiratory viruses such as influenza [16]. It has been proposed that changes in the composition of the intestinal microbiota may negatively impact lung function through systemic immunological effects, whereas lung inflammation in the context of respiratory viral infections may lead to gut dysbiosis [17]. The interrelation and bidirectional effect between the microbiota of the aforementioned anatomical compartments is referred to as the gut-lung microbiota axis. Previous studies have shown that alteration in the composition of the intestinal microbiota, through probiotic or antibiotic administration, affects the outcome of respiratory viral infections such as influenza [18], whereas commensal bacteria in the respiratory tract may be able to strengthen the mucosal immunity of the respiratory tract [19]. Similar mechanisms have been proposed with the pathogenesis of COVID-19 infection [20]. In particular, the gut microbiota may play a role in regulating the ACE2 receptor which binds to SARS-CoV-2 [20, 21], while the respiratory microbiota may protect against infection with SARS-CoV-2 by enhancing respiratory tract immune responses [22]. This scoping review aims to identify the latest evidence derived from primary research studies investigating the possible alteration of gut and/or airway microbiota during infection with SARSCoV-2. Understanding of the mechanisms involved in this association will support the development of effective strategies to diagnose, manage and prevent COVID-19 disease.

\section{Methods}

This scoping review was conducted based on the methodological framework developed by key authors in the field. All the steps involved comply with the most recent relevant guidance [23, 24].

\section{Review questions}

1. Is there an association between infection with SARS$\mathrm{CoV}-2$ and changes in the gut and/or airway microbiota?

How does the gut and/or airway microbiota of COVID-19 patients compare with the microbiota of patients infected with other viruses and healthy participants?

2. What is the association between the gut and/or airway microbiota, the immune system and COVID-19 pathogenesis and disease severity?

3. Is there any evidence for a potential benefit of using faecal microbiota transplantation (FMT) on COVID-19 patients during their recovery?

\section{Search strategy}

A search on Pubmed, Science Direct, DOAJ and Cochrane databases until 14 May 2021 was conducted by researcher DL and validated by researcher KR. Examples of search terms used on Pubmed are listed in Supplementary Material 1 , while search terms were adapted in other databases as appropriate.

\section{Inclusion criteria}

Included studies were observational studies or clinical trials assessing the association between gut and/or airway microbiota and COVID-19 infection in human participants (Table 1).

\section{Exclusion criteria}

Non-human, non-English studies and in-progress clinical trials were excluded. 
Table 1 Inclusion criteria of studies included in scoping review

\begin{tabular}{ll}
\hline Population & COVID-19 patients \\
\hline Interest & Patients' changes of airway and/or gut microbiota composition \\
Comparison & Healthy individuals or patients with other viruses \\
Outcome & Type of microbiome detected after COVID-19 infection \\
Study type & Quantitative method \\
\hline
\end{tabular}

\section{Data extraction}

Data extraction was carried out by researcher DL and validated by researcher KR. The following data were extracted: author name, type of study, country in which the study was conducted, sample size, median age of the participants in the study, study objective, whether and which serum inflammatory markers were evaluated, whether any antimicrobials or probiotics were administered to the participants of the study, sample collection and evaluation methods and key findings (including microbiota affected).

\section{Data synthesis}

The process of data synthesis involved summarising the key findings of the included studies and exploring the relationship between studies in a narrative form. In addition, the mean and the SEM of any numeric figures were calculated. The studies were grouped into studies investigating: (a) gut microbiota (Table 2) or (b) airway microbiota (Table 3) and (c) both gut and airway microbiota (Table 4).

\section{Results}

\section{Overview of included studies}

\section{Study selection}

The PRISMA flow diagram [25] shows the number of studies identified, studies excluded and final studies included in the current review paper (Fig. 1). The initial search retrieved 312 articles through database search and two extra papers were found through manual checking of other literature reviews. 44 articles were eliminated due to duplication, giving rise to 270 articles. After screening the papers by reviewing the abstracts and titles and applying the inclusion and exclusion criteria, 35 papers were eligible to be included in this review. During the full manuscript review process, 13 papers were deemed ineligible since they were not relevant to our current research question ( 2 of them did not include any COVID-19 patients or investigate SARS-CoV-2 virus, 5 of them were animal studies, 3 of them were not observational studies or clinical trials and 3 of them did not investigate the direct relationship between COVID-19 and airway or gut microbiota). In total, 22 studies were included in the review.

\section{Study characteristics}

Ten of the 22 studies included in the review investigated the association between COVID-19 infection and the composition of the gut microbiota, 11 studies examined the changes in the composition of the airway microbiota during infection with COVID-19 and one study focused on the association between COVID-19 and both the airway and gut microbiota. The main characteristics of the studies are shown in Tables 2, 3, 4. Ten studies investigated the association between gut microbiota and COVID-19 [26-35], 11 studies investigated the association between airway microbiota and COVID-19 [36-46] and one study investigated the association between both gut and airway microbiota and COVID-19 [47]. All studies collected their samples after onset of infection with SARS-CoV-2.

Studies investigating the association between gut microbiota and COVID-19 Among the 10 studies investigating the association between gut microbiota and COVID-19 [26-35], eight studies were cohort studies [27-33, 35], one study was a cross-sectional study [26], and one study was a clinical trial [34] (Table 2).

Nine studies focused on the intestinal dysbiosis during COVID-19 infection [26-33, 35], 4 studies examined the relationship between COVID-19 severity with gut microbiota composition [27, 30, 32, 35]. 2 studies focused on intestinal dysbiosis post COVID-19 infection [28, 33] and one study assessed the efficacy of faecal microbiota transplantation post COVID-19 infection [34]. Eight studies collected the microbiota DNA from stool samples [26-31, $34,43]$ while 2 studies collected their samples from rectal swabs $[32,35]$. Five studies included participants who were administered antimicrobials and/or probiotics prior to sample collection [27, 28, 30, 32, 35], 3 studies excluded these participants $[26,31,32]$ while 2 studies did not report this information [29, 33]. Six studies had recruited their participants from China [26, 27, 31-34], three studies from Hong Kong [28-30] and one from Italy [35]. The mean sample size of all studies was $62(\mathrm{SEM}=16)$ and the median age of COVID-19 subjects was 55.09 (SEM=3.41). Only one study [35] mentioned the sample collection time with 


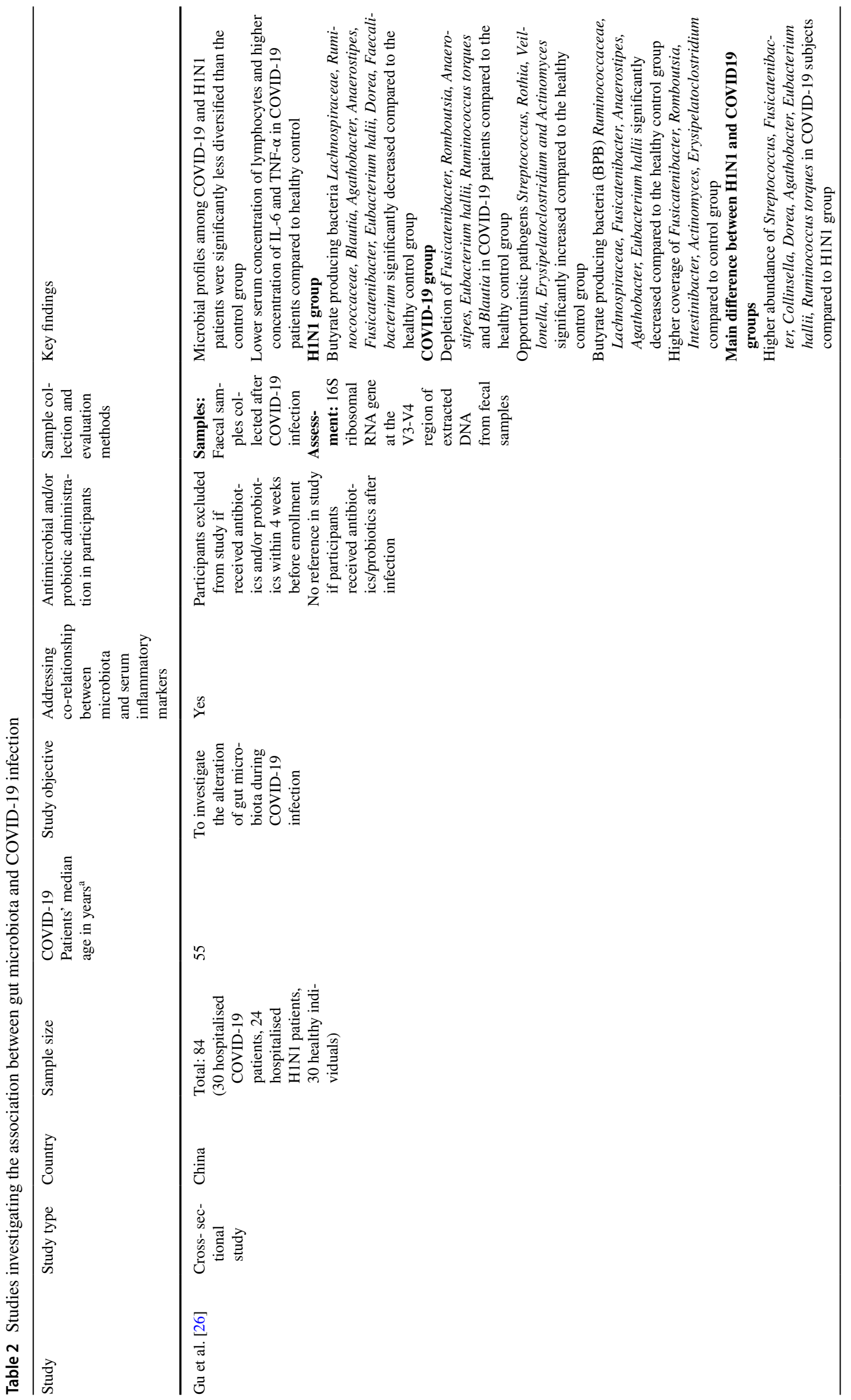




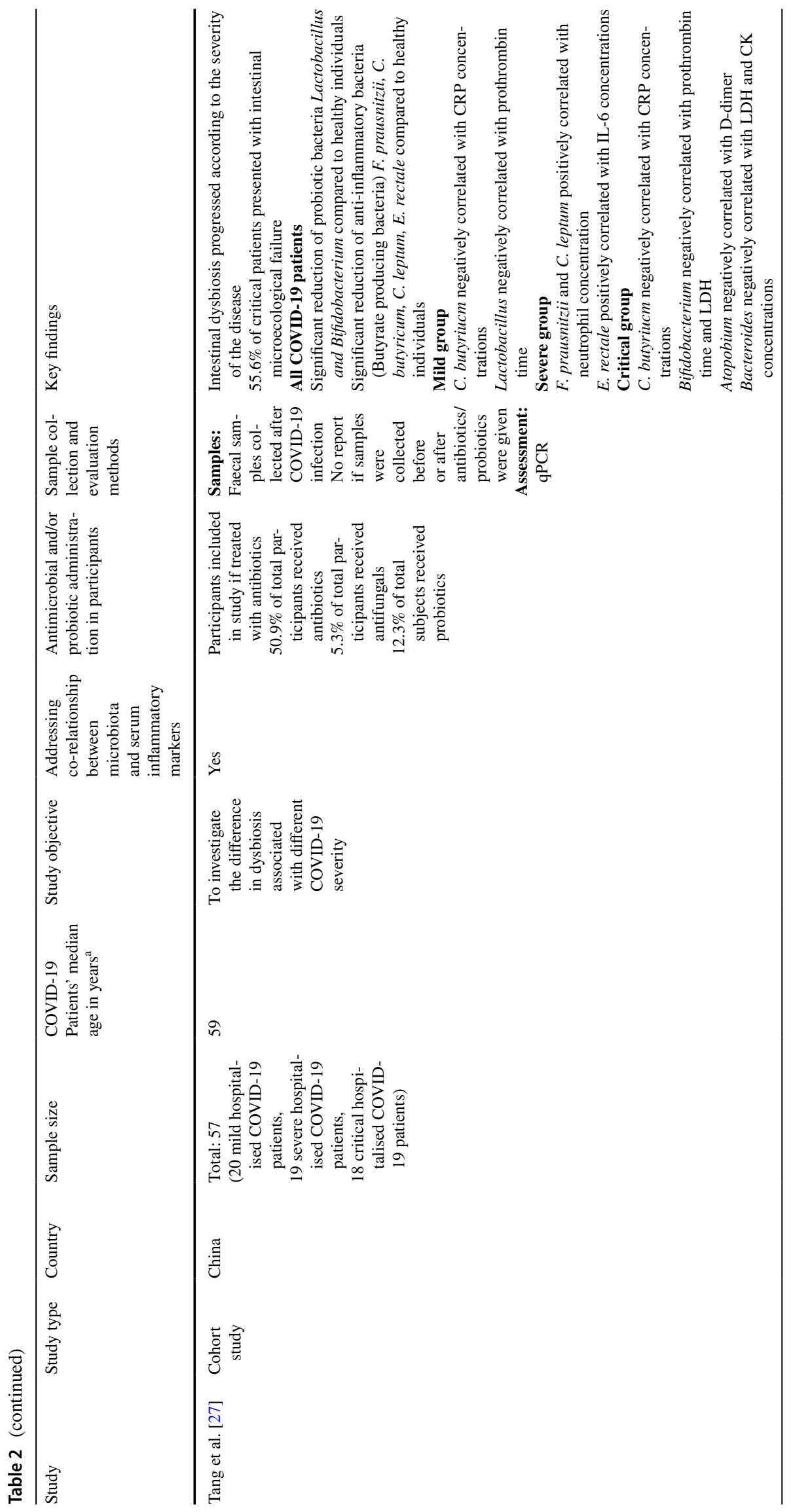




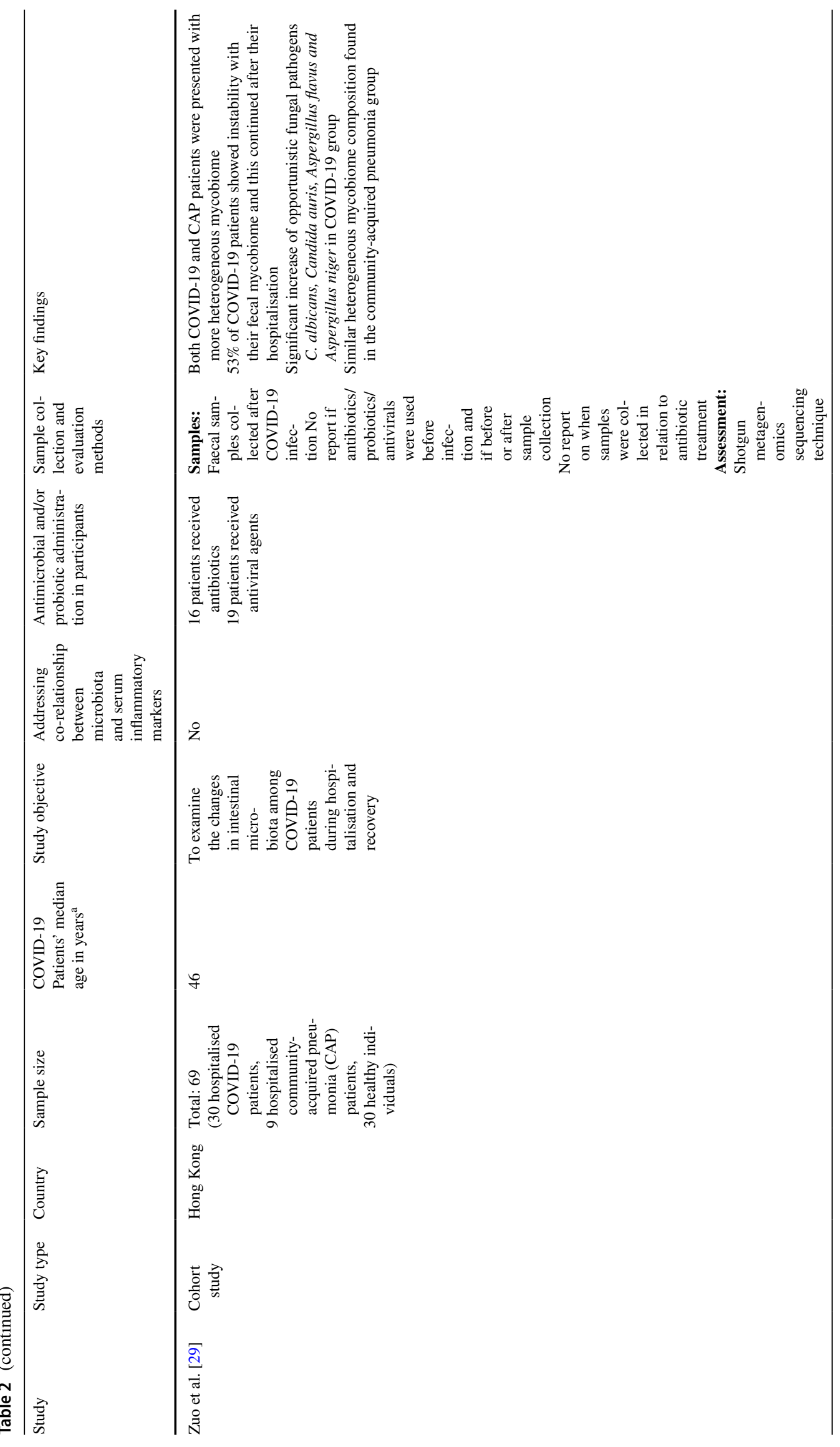




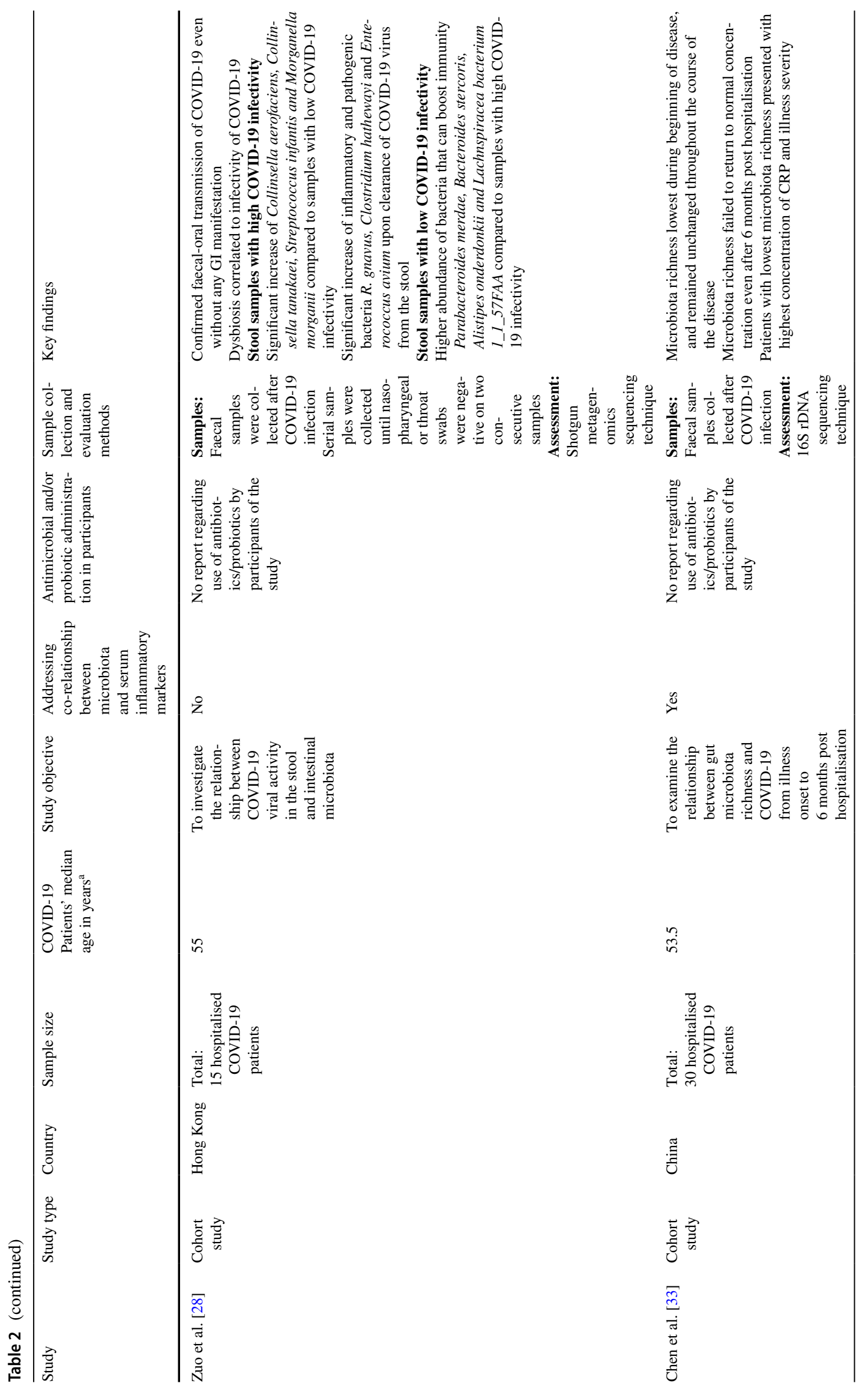




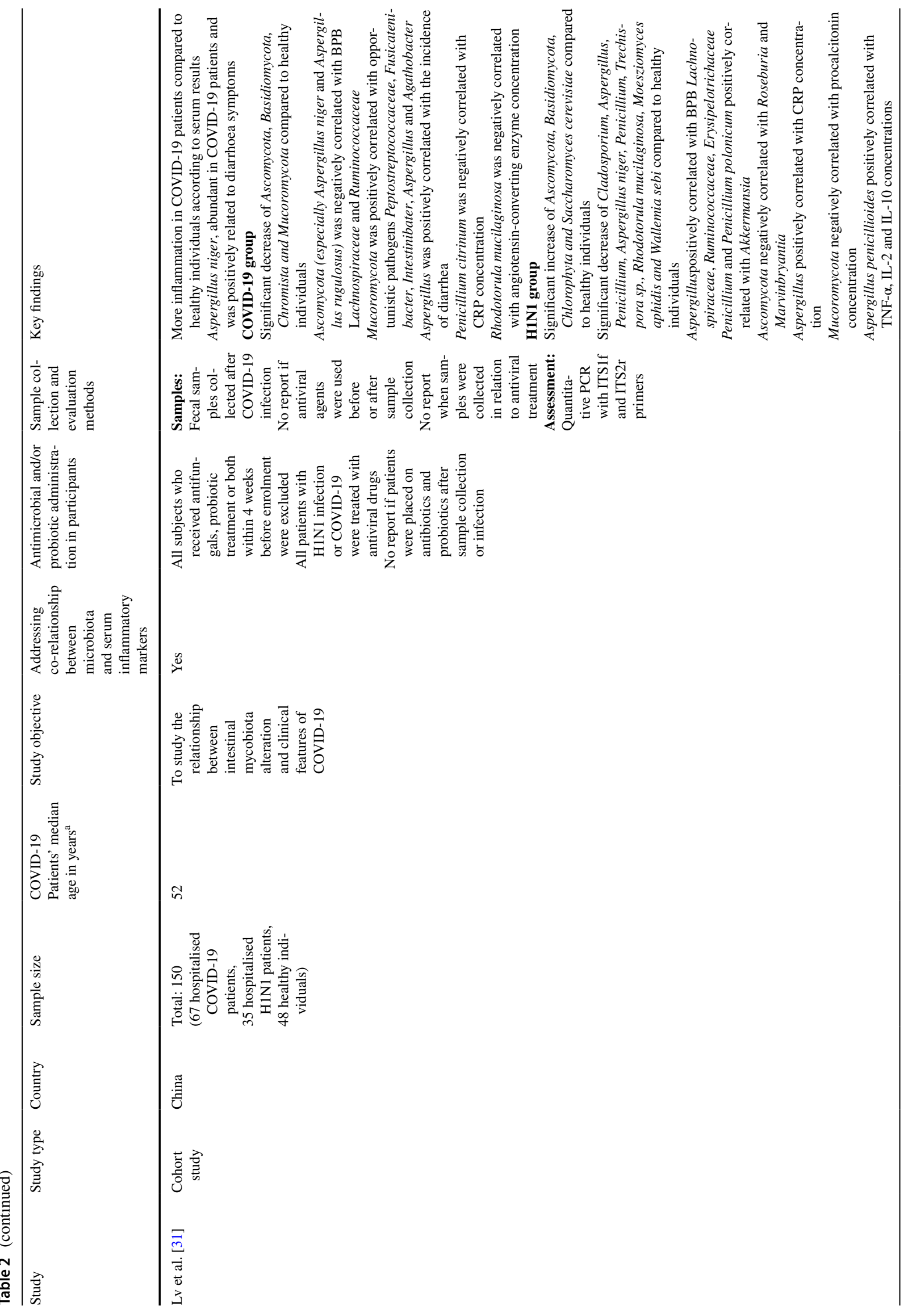




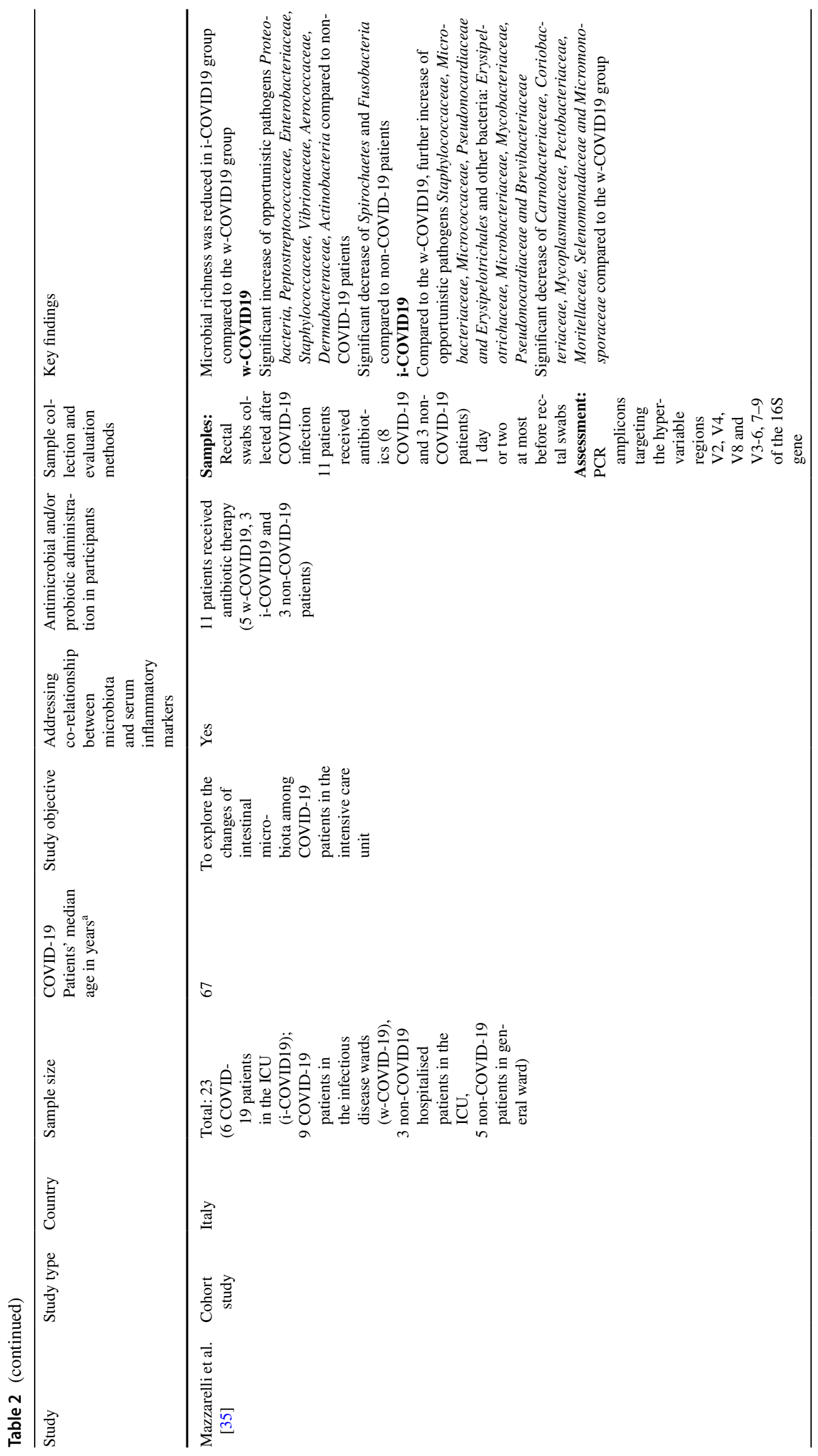




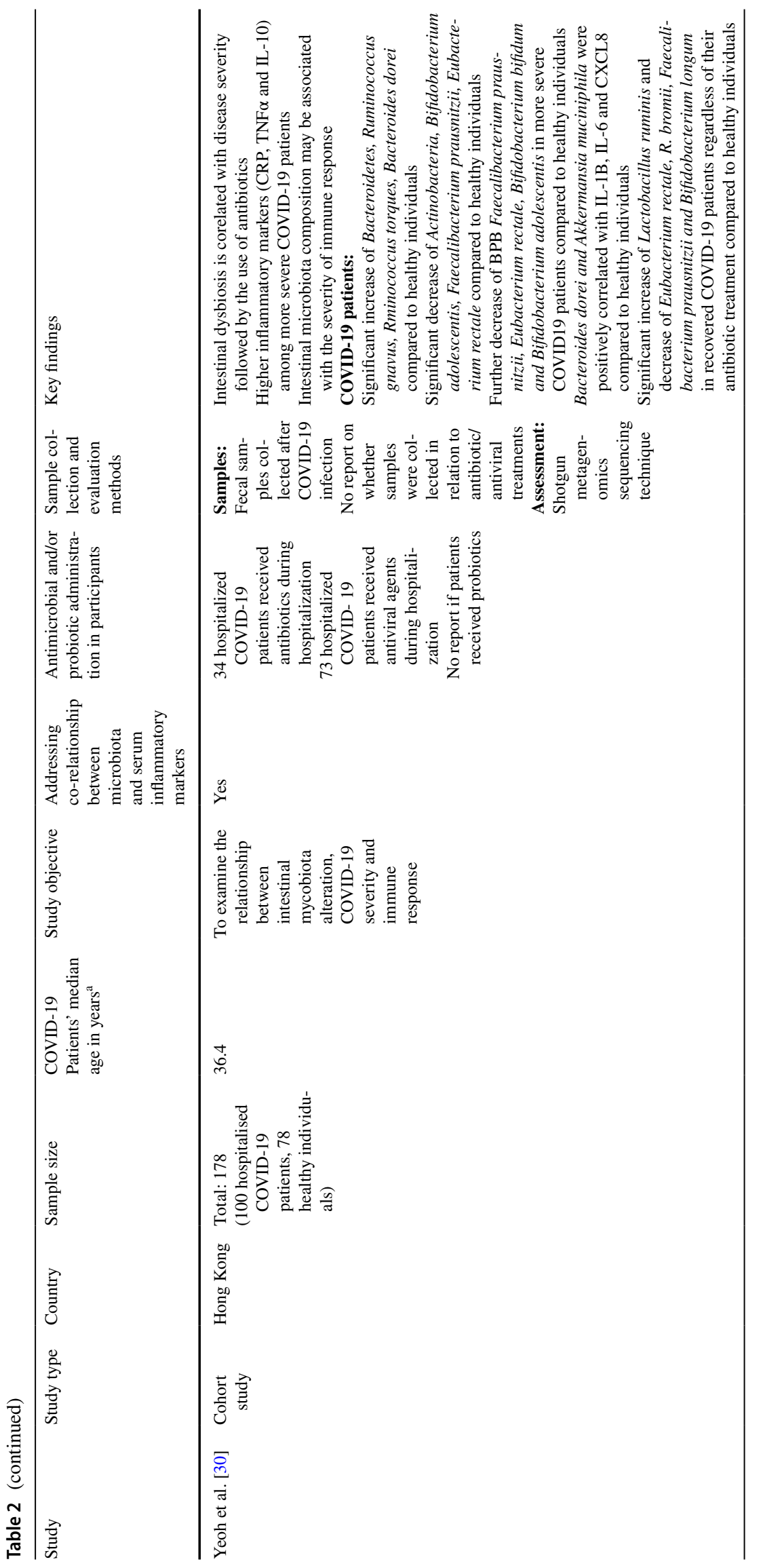




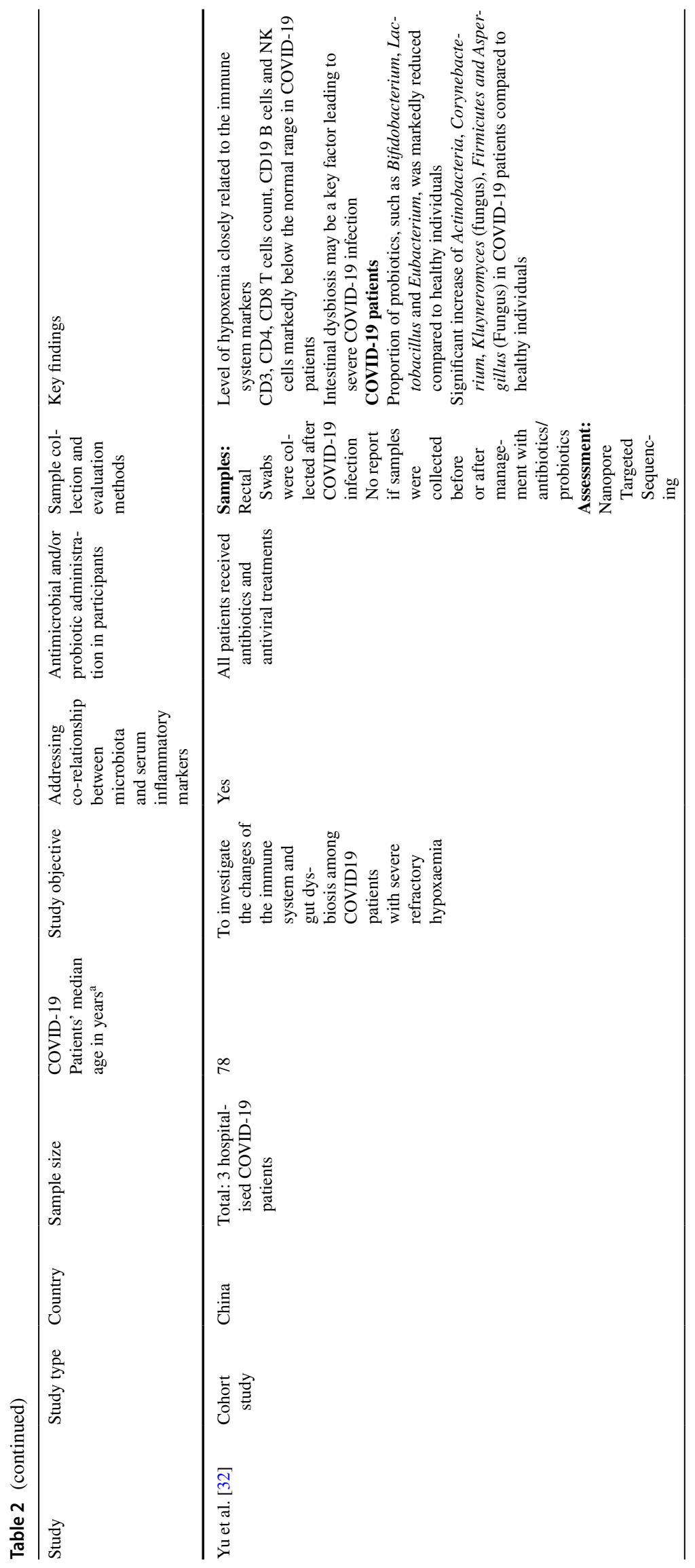




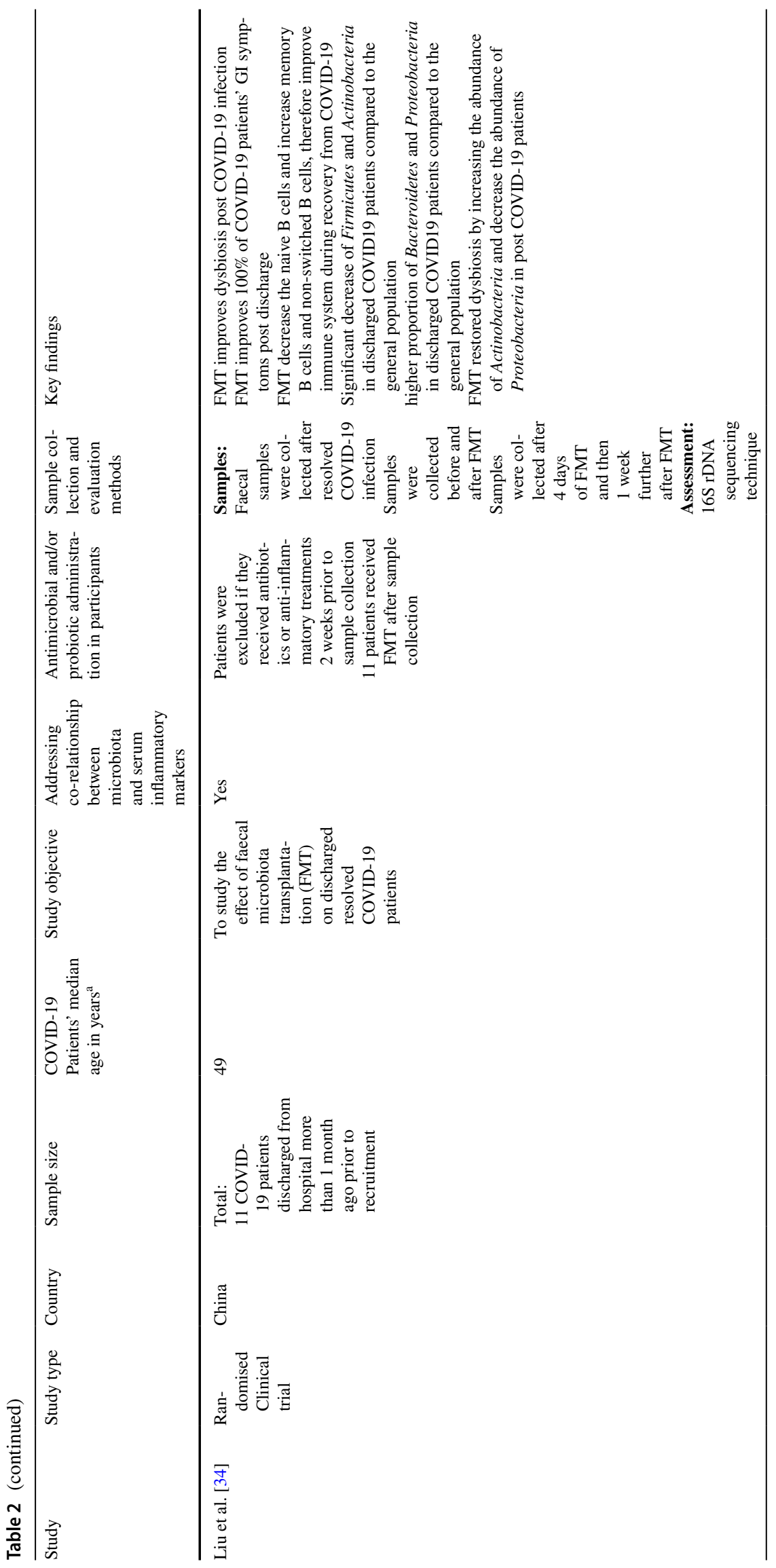




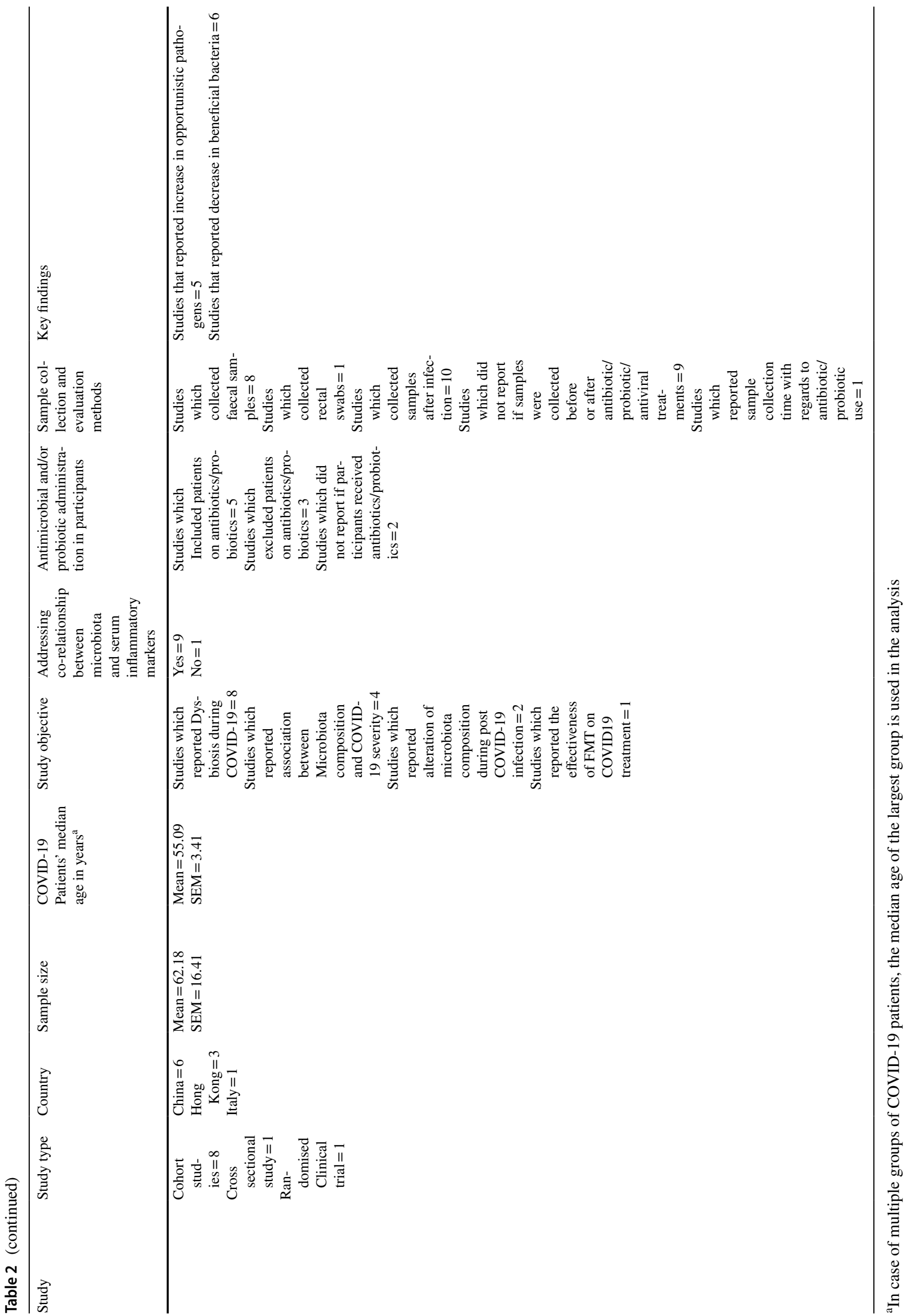




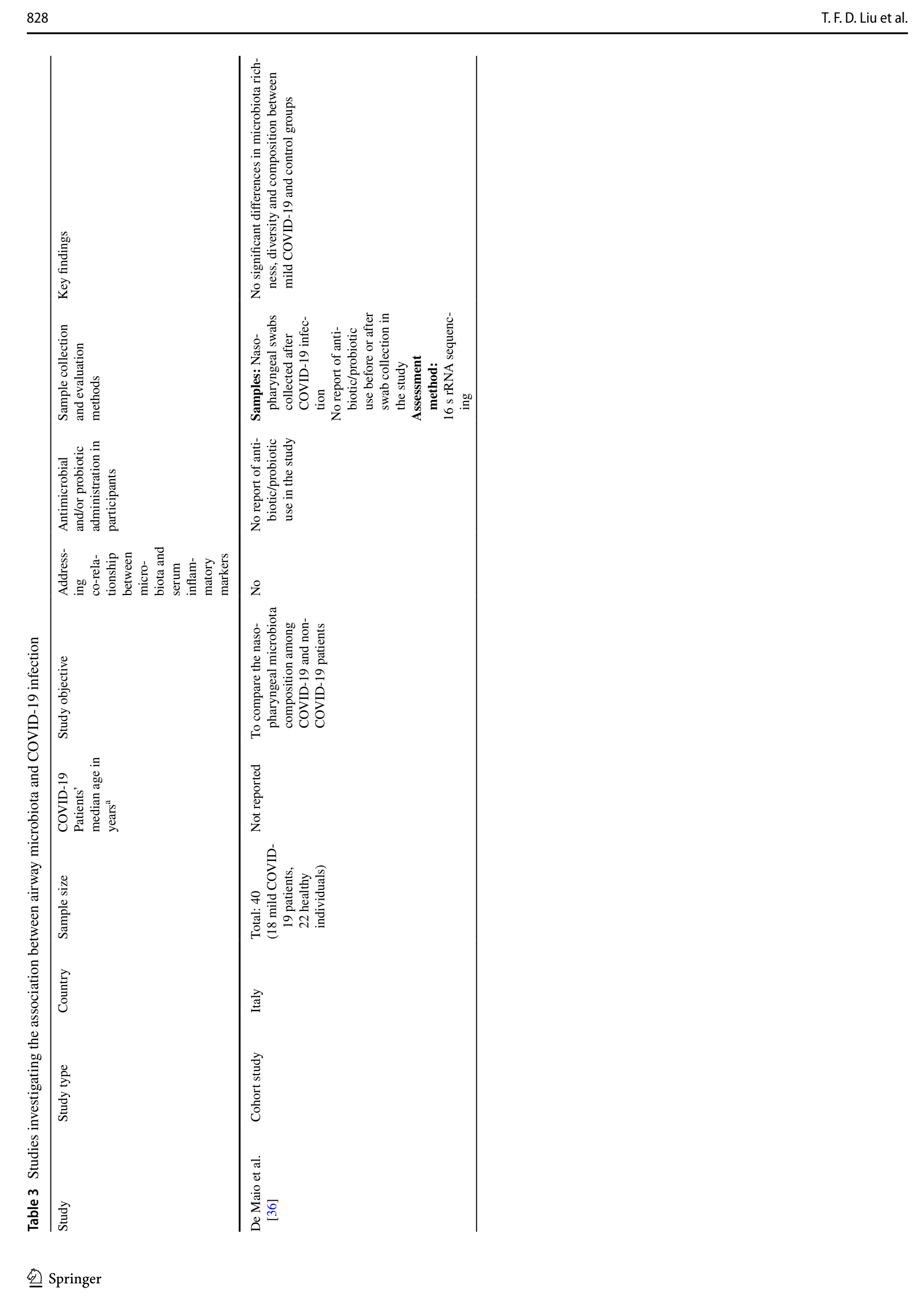




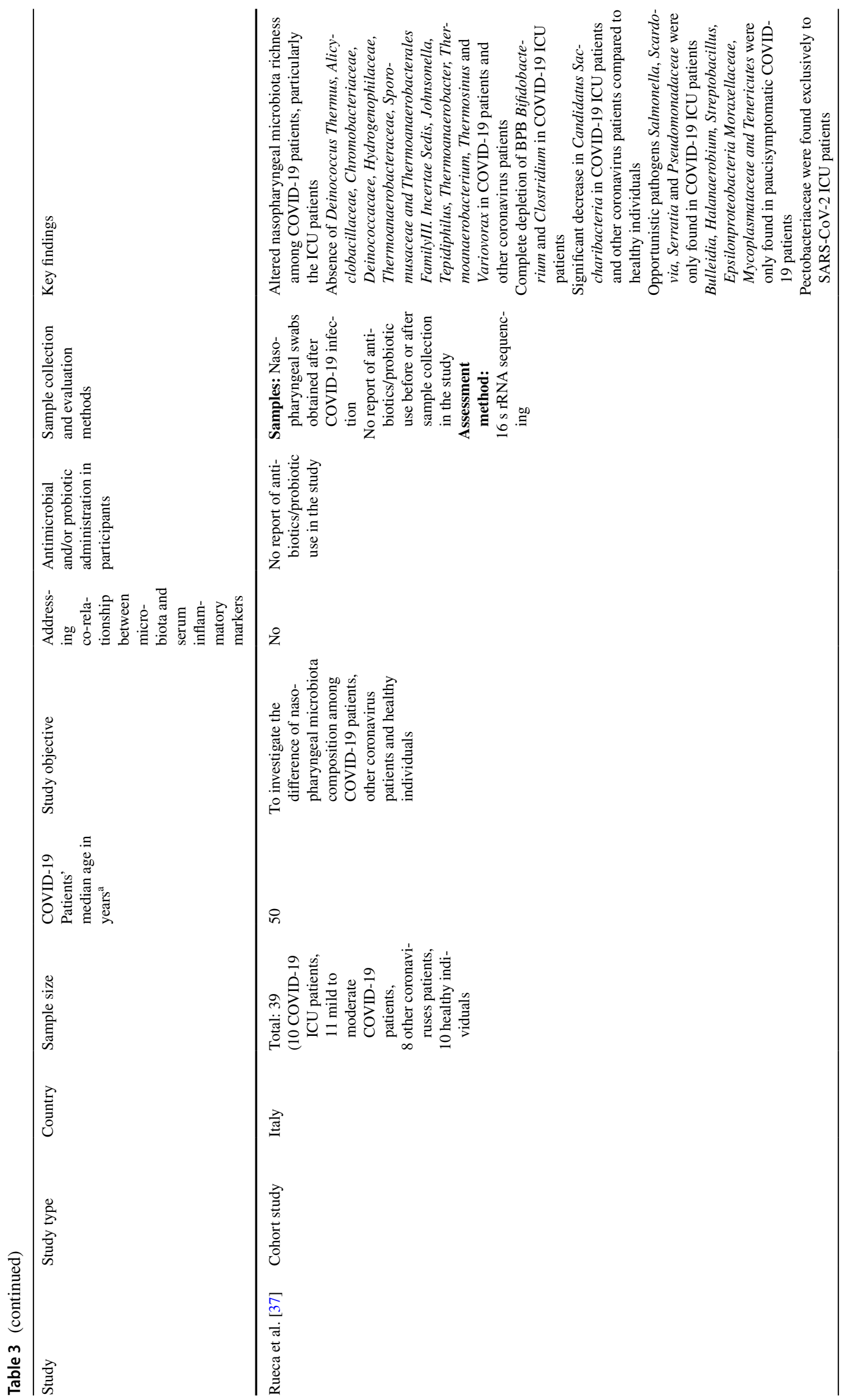




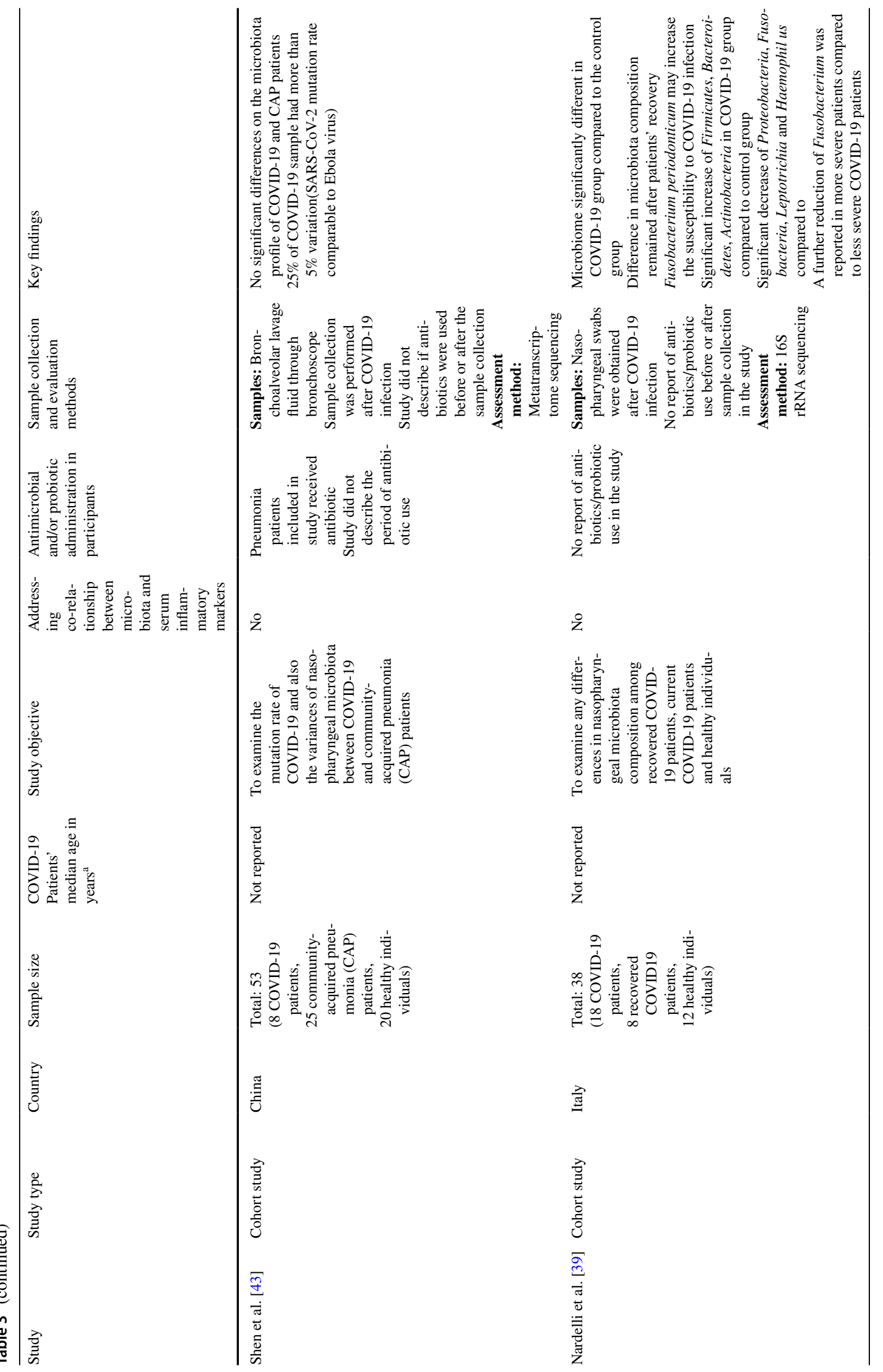




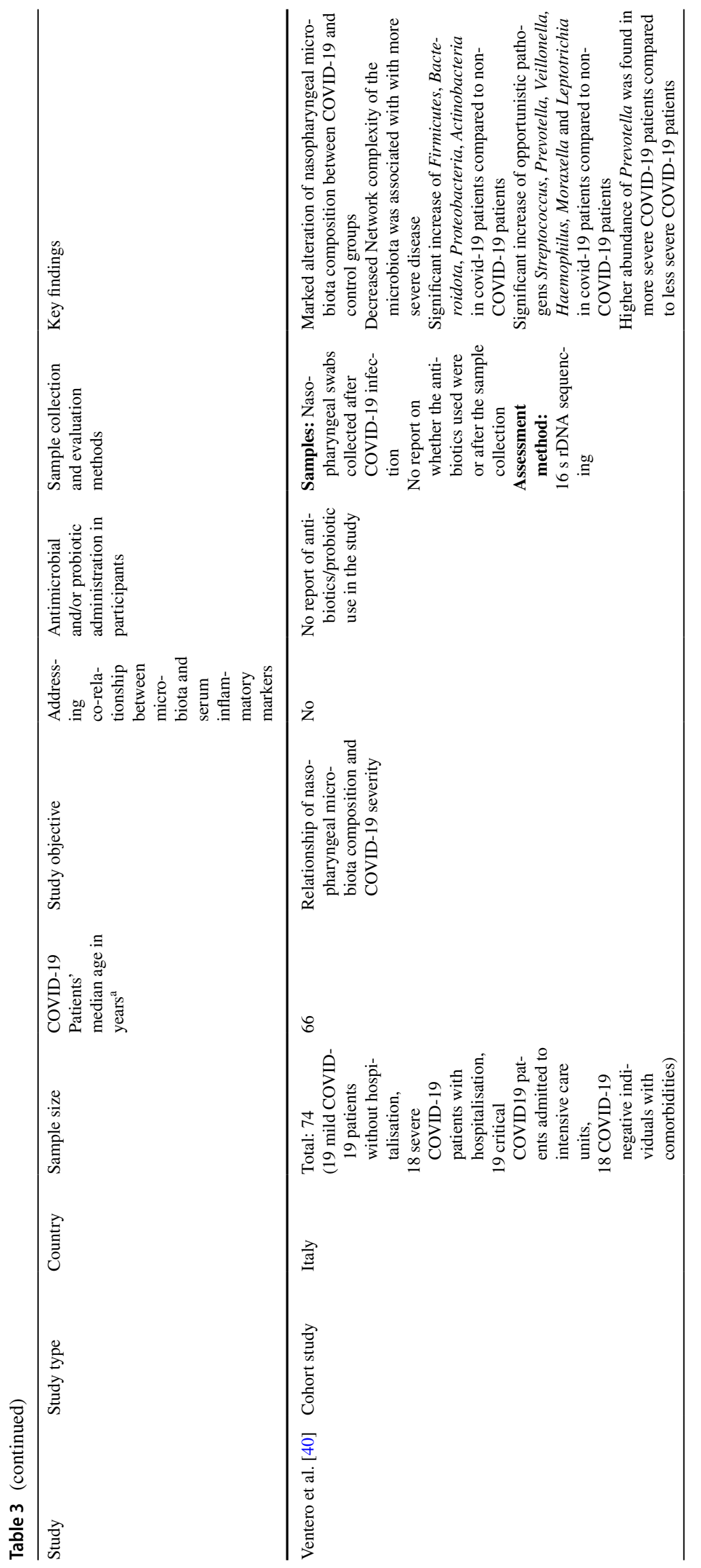


832

T. F. D. Liu et al.

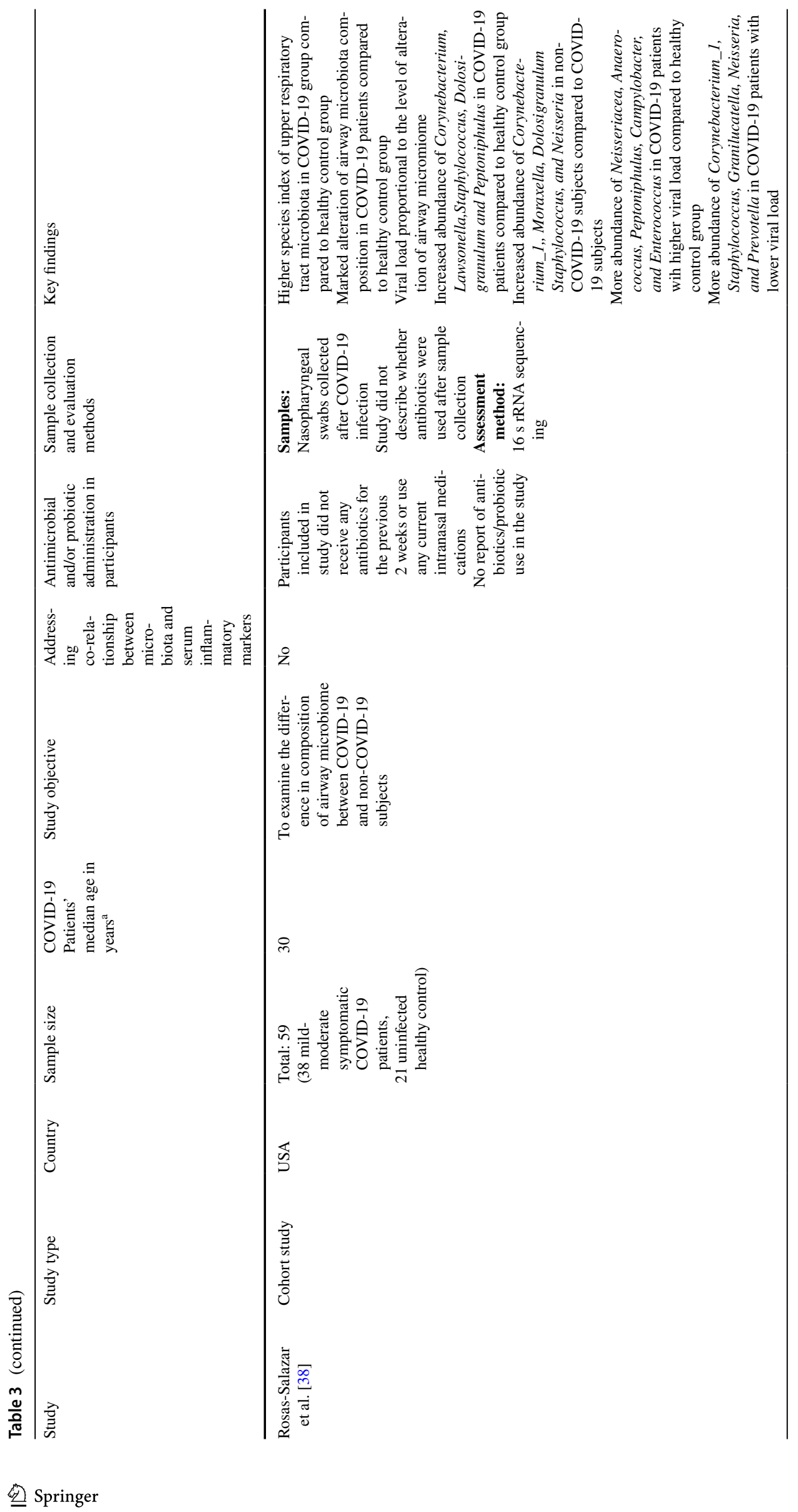




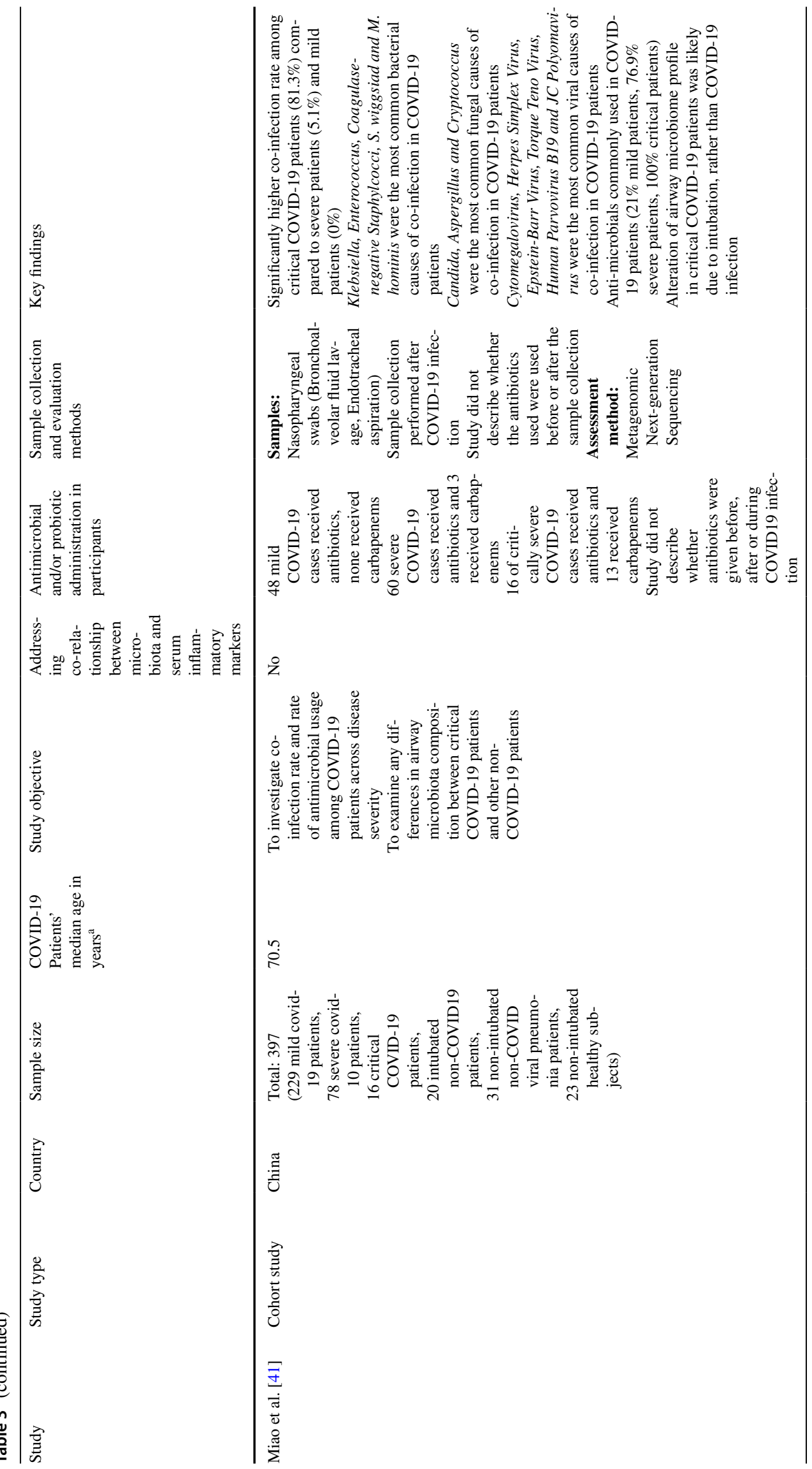




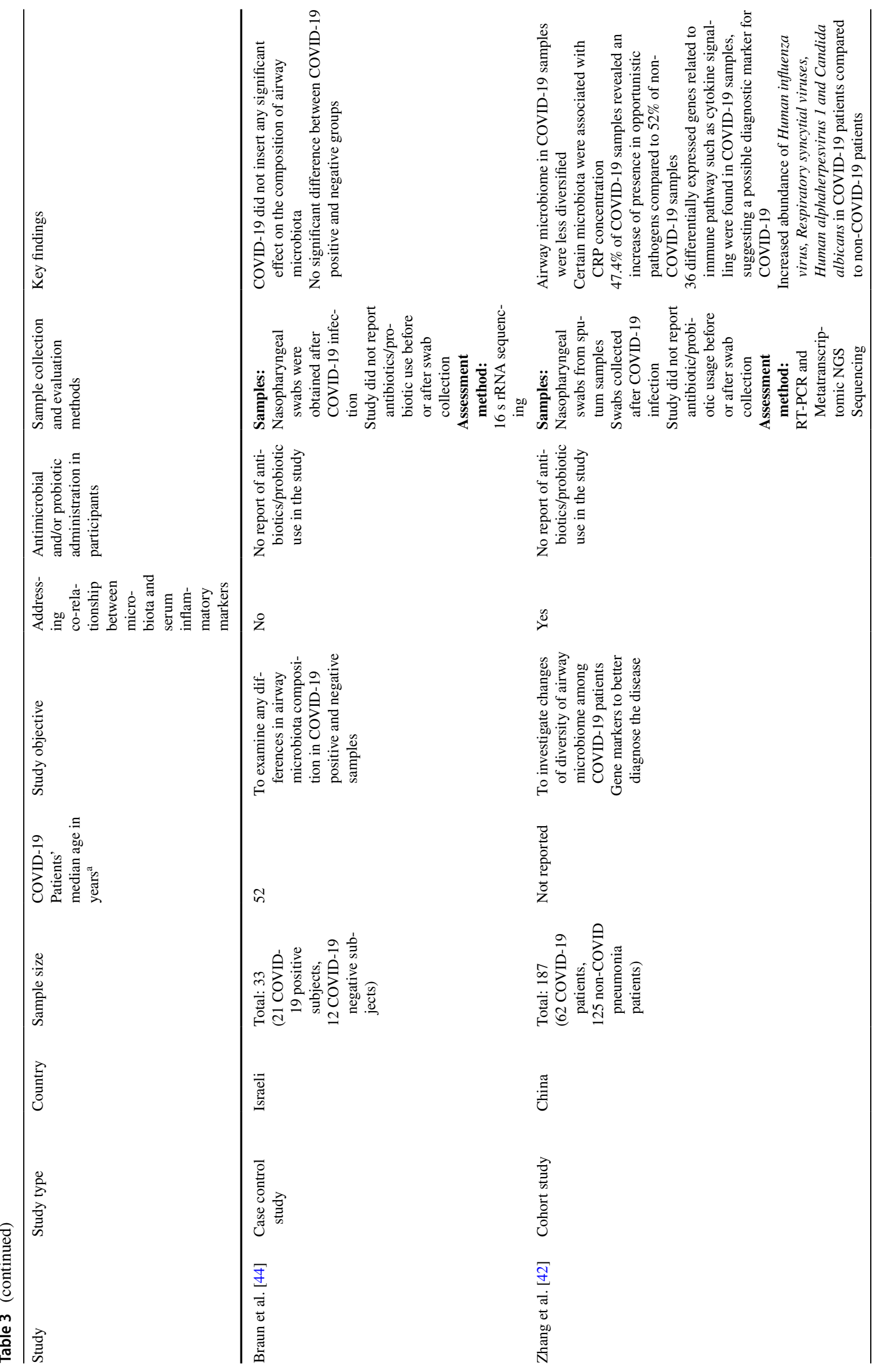




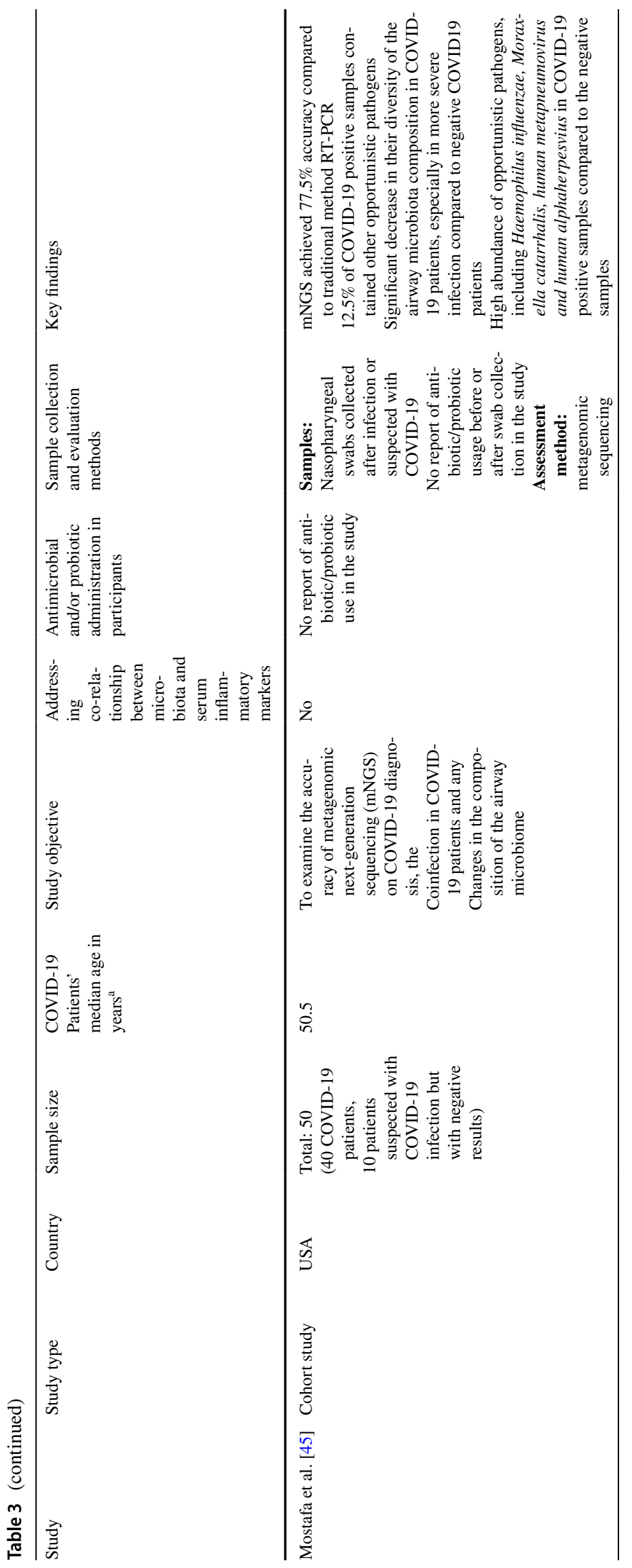


836

T. F. D. Liu et al.

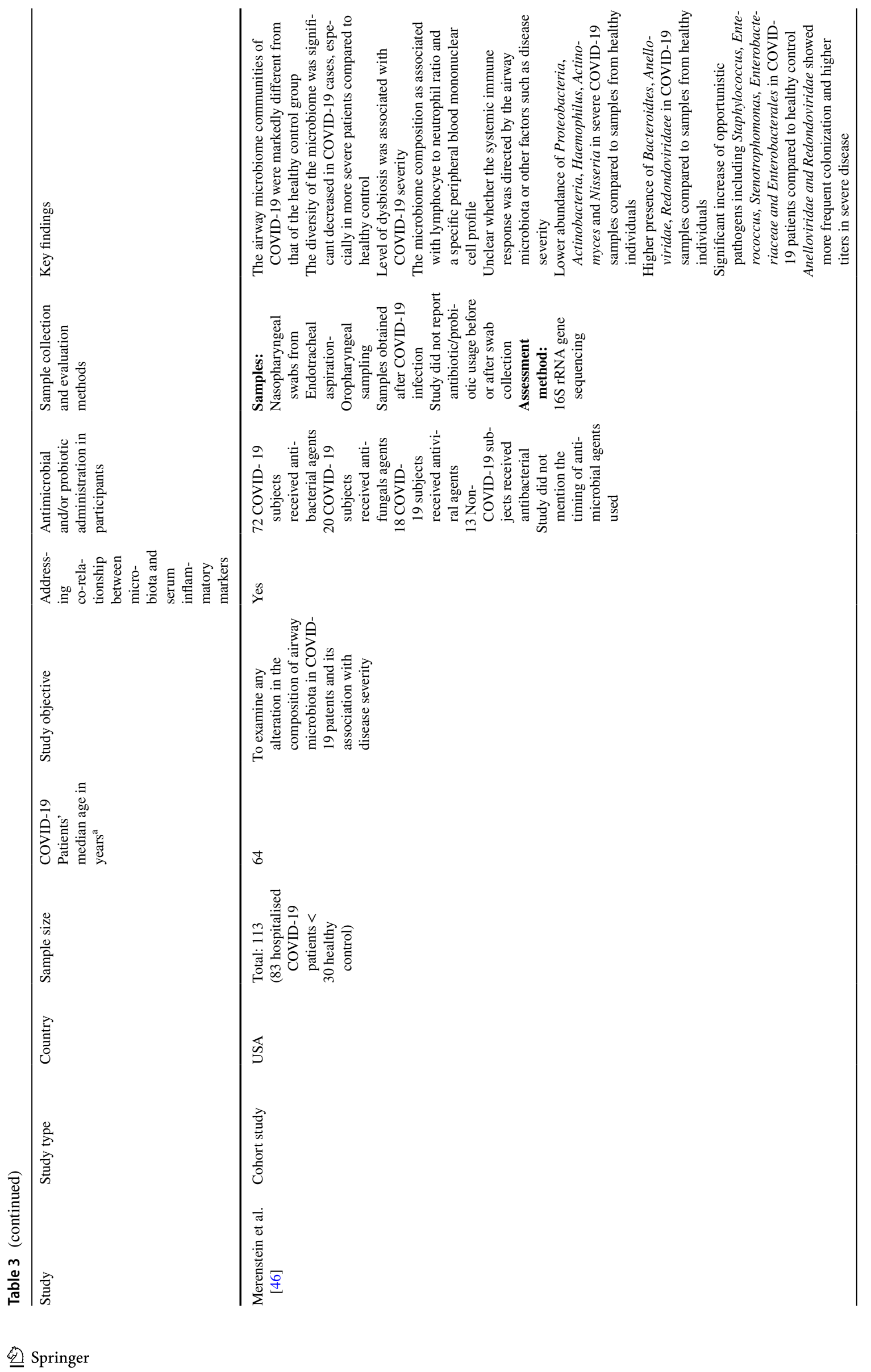




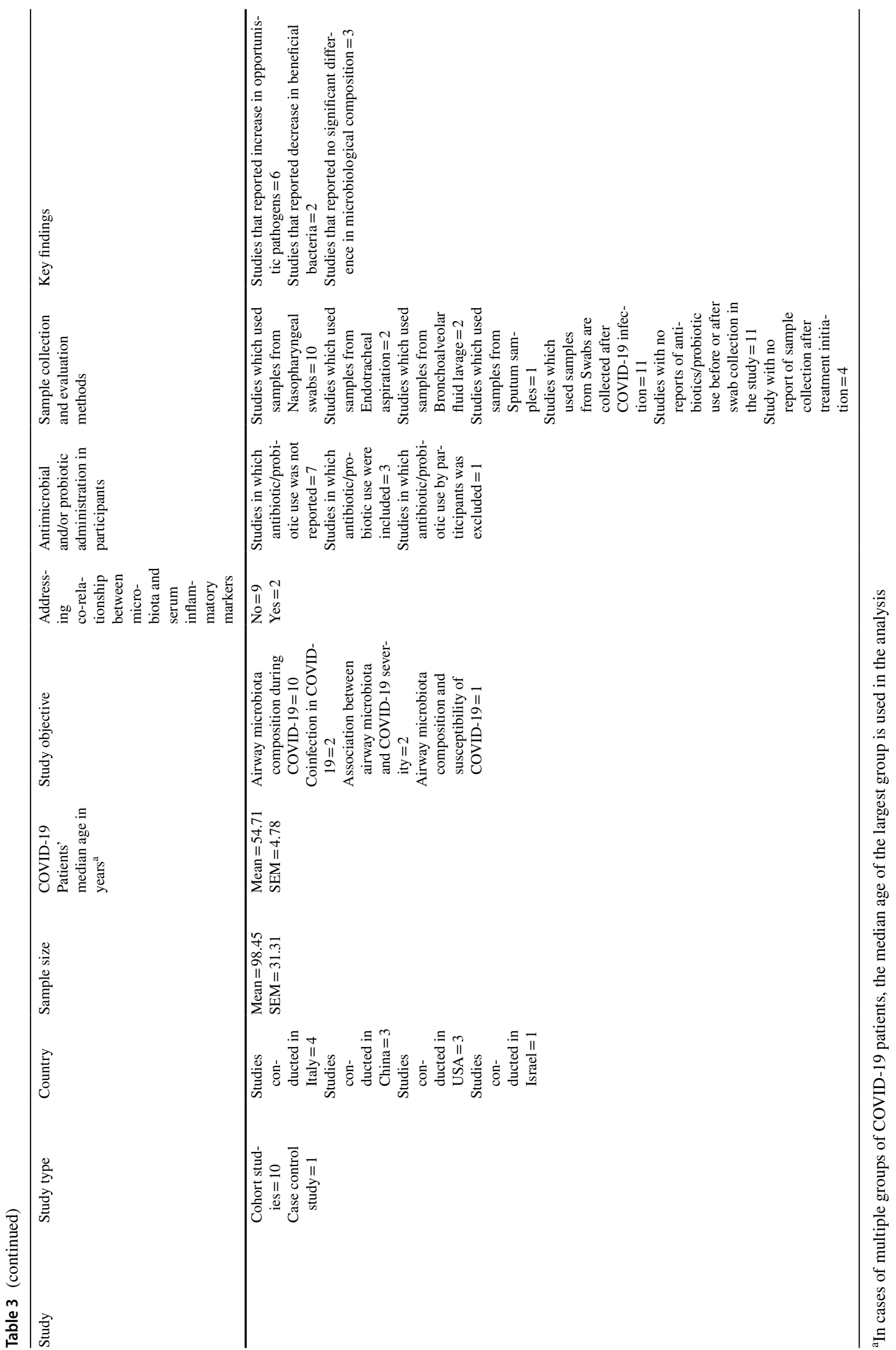




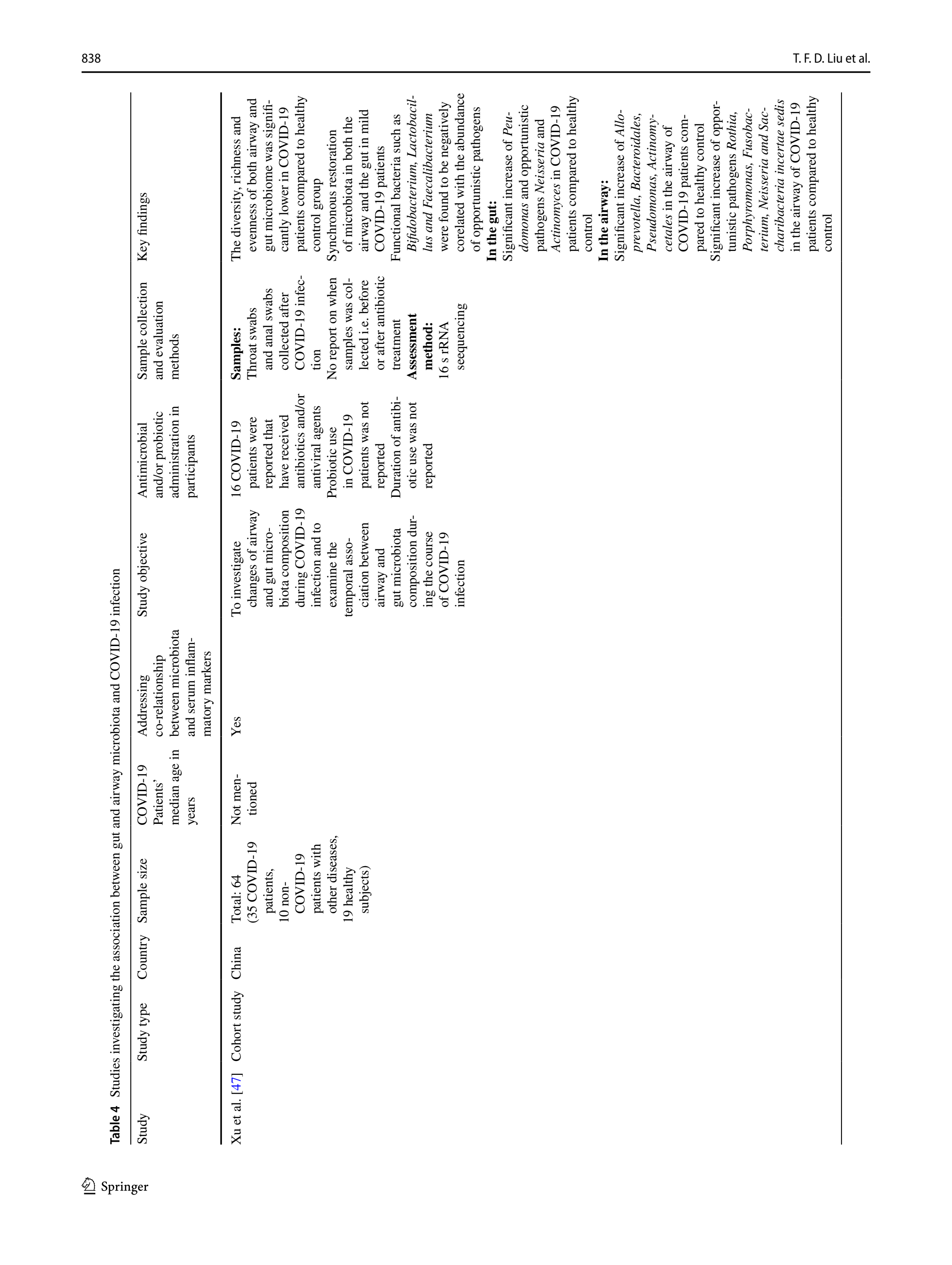


regards to antibiotic/probiotic/antiviral use; all other studies did not disclose this information.

\section{Studies investigating the association between airway micro-} biota and COVID-19 The search identified 10 cohort studies [36-43, 45, 46] and one case-control study [44] investigating the association between airway microbiota and COVID19 (Table 3).

Eleven studies investigated the composition of the airway microbiota during COVID-19 infection [36-46], 2 studies examined the association between the composition of airway microbiota and COVID-19 severity [40, 46] and 2 studies assessed the prevalence of co-infection during COVID-19 $[41,45]$. Ten studies examined the DNA of the microbiota collected from nasopharyngeal swabs [36-42, 44-46], 2 studies collected DNA samples from endotracheal aspiration $[41,46]$ and 2 studies collected samples using bronchoalveolar lavage [41, 43]. Three studies included participants who were administered with antimicrobials and/or probiotics prior to sample collection $[41,43,46]$, one study excluded these participants [37] and 7 studies did not report this information [36, 37, 39, 40, 42, 44, 45]. None of the studies reported if antibiotics or probiotics were used before or after the sample collection.

Subjects were recruited from Italy in four studies [36, 37, 39,40 ], from China in three studies [41-43], from the US in three studies [38, 45, 46] and from Israel in one study [44]. The mean sample size of all studies was $98(\mathrm{SEM}=31)$. The average median age of the COVID-19 subjects in the studies was $54.71(\mathrm{SEM}=4.78)$.

Studies investigating the association between both gut and airway microbiota and COVID-19 Only one study investigated the association between both the gut and airway microbiota and COVID-19 [47]. Sixty-four participants were recruited to study the temporal changes of airway and gut microbiota composition during COVID-19 infection. Both anal and throat swabs were used to collect the samples, while subjects had received antimicrobial treatment prior to sample collection (Table 4).

\section{Study outcomes}

\section{Studies investigating the association between gut microbiota and COVID-19}

Among the 10 studies investigating the role of the gut microbiota on COVID-19, 5 studies concluded that COVID-19 infection increased the amount of opportunistic pathogens in the gut $[26-29,31]$ and 6 studies suggested that COVID-19 infection reduced the number of beneficial gut microbiota [26, 27, 29-32] (Table 2).
Comparison of gut microbiota in COVID-19 patients vs. patients infected with other viruses and healthy participants $\mathrm{Gu}$ et al. [26] examined the difference in composition of gut microbiota of COVID-19 patients compared to patients infected with $\mathrm{H} 1 \mathrm{~N} 1$ and healthy individuals due to the similarities of clinical presentation and transmission routes of H1N1 and SARS-CoV-2. The microbial profiles of the COVID-19 and H1N1 patients were significantly less diversified than those of the healthy control group. Moreover, even though the clinical presentations of COVID-19 group and H1N1 group were similar, a lower plasma concentration of lymphocytes and a higher concentration of IL- 6 and TNF- $\alpha$ were found in the COVID-19 patients compared to the healthy control group [26] (Table 2).

$\mathrm{Lv}$ et al. [31] investigated the association between COVID-19 clinical features and intestinal microbiota, and they found an association between Aspergillus niger and diarrhea symptoms, which was present in $20.89 \%$ of COVID-19 patients in this study [31] (Table 2).

Zuo et al. [29] investigated the changes in intestinal fungal microbiota among patients with COVID-19 during their hospitalisation and their recovery. The results of the study showed that the COVID-19 group and healthy control group demonstrated a similar faecal mycobiome profile at baseline; however, patients with COVID-19 and community-acquired pneumonia (CAP) presented with high mycobiome heterogeneity during the course of the disease. Fifty-three percent of COVID-19 patients showed instability with their fecal mycobiome and this continued after their discharge [29].

Gut microbiota, the immune system and disease severity $\mathrm{Yu}$ et al. [32] described the changes of the immune system and gut dysbiosis among 3 COVID-19 patients with severe refractory hypoxaemia and reported a different gut microbiota composition in COVID-19 patients compared to that of healthy individuals. The researchers concluded that the intestinal dysbiosis may be a key factor influencing COVID-19 severity [32] (Table 2).

Yeoh et al. [30] compared the stool samples from hospitalised COVID-19 patients with non-COVID-19 healthy individuals to investigate if intestinal microbiota ecology can reflect COVID-19 clinical severity and immunological profile. It was reported that intestinal dysbiosis being the most important contributing factor for disease severity, while the use of antibiotics was the second most important factor. More importantly, as higher inflammation markers, including CRP, TNF- $\alpha$ and IL-10, were found among COVID-19 patients with severe disease, it was proposed that the alteration of intestinal microbiota composition may be associated with hyper-inflammatory responses [30].

Zuo et al. [28] detected a high level of COVID-19 genome in 7 out of 15 patients' stool samples, while none of these patients presented with any GI symptoms. A higher 


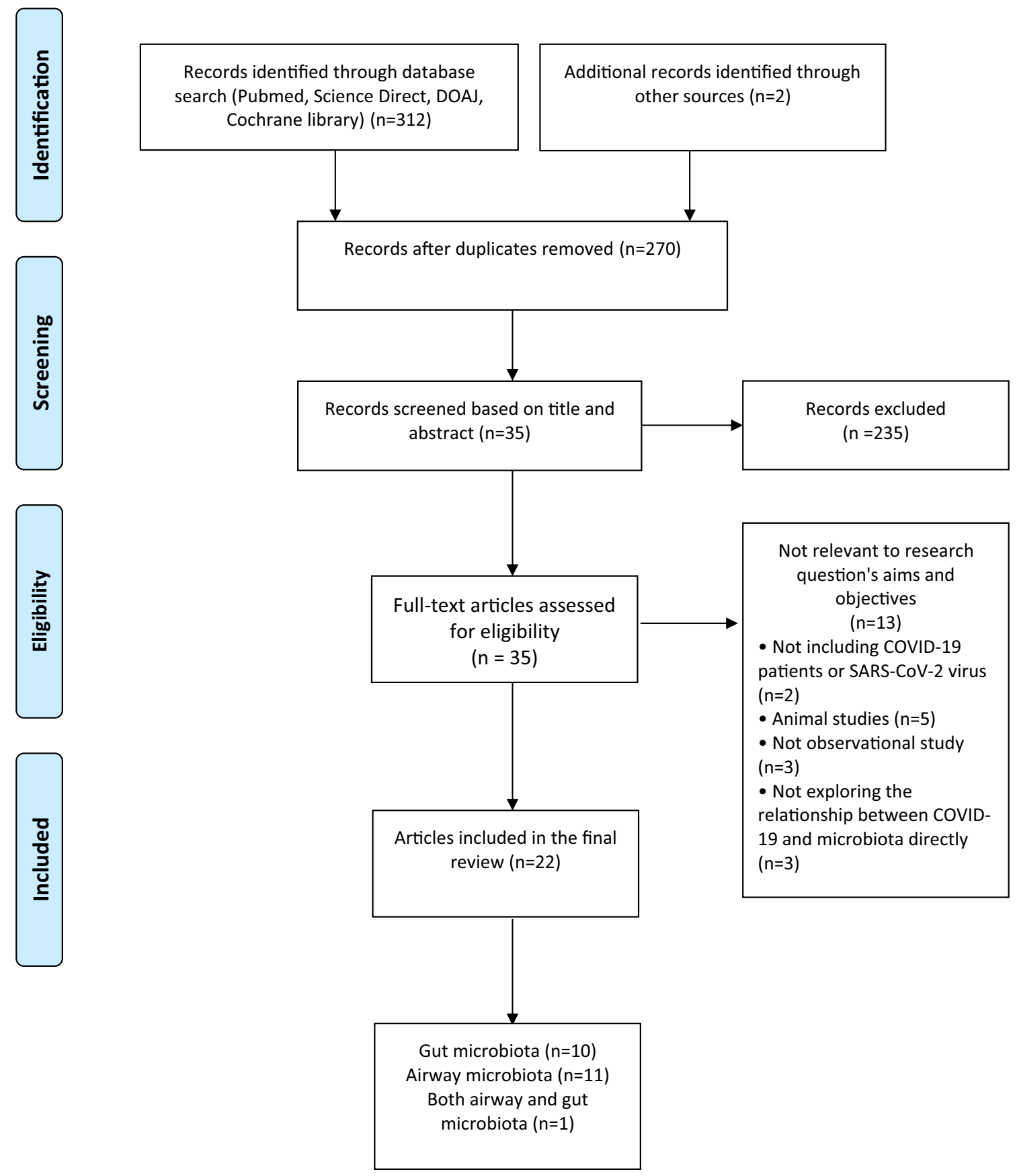

Fig. 1 PRISMA flow diagram on the selection of studies included in the current scoping review

abundance of opportunistic bacterial species were found among the stool samples with high COVID-19 infectivity. On the other hand, bacteria associated with immune priming, such as butyrate-producing bacteria, were found in the faecal samples of patients with low SARS-CoV-2 infectivity. These results highlight the importance of the potential faecal-oral transmission route even in patients without GI manifestations [28].
Tang et al. [27] recruited COVID-19 hospitalised patients and categorised them by disease severity. Analysis of stool samples showed a decreased abundance of beneficial bacteria and an increased abundance in opportunistic pathogens in all COVID-19 patients. The intestinal dysbiosis progressed according to the severity of the disease and $55.6 \%$ of critical patients presented with intestinal micro-ecological failure. Based on these findings, it was suggested that the intestinal 
microbial profile could be used as a diagnostic biomarker and a prognostic factor of the severity of COVID-19 [27].

Chen et al. [33] conducted a 6-month follow-up study to determine the diversity of the intestinal microbiota among COVID-19 patients during different timepoints of the disease. It was concluded that the microbiota richness was reduced after disease onset; microbiota diversity decreased during the beginning of the disease and remained unchanged throughout the course of the disease. Interestingly, microbiota richness failed to return to normal even after 6 months post hospitalisation. Patients with the lowest diversity presented with the highest CRP concentration and disease severity, therefore indicating a potential association between the extension of intestinal dysbiosis, inflammatory response and clinical severity [33].

By comparing COVID-19 patients in the intensive care units (ICU) and the infectious diseases wards (ID), Mazzarelli et al. [35] found that microbial richness was reduced in the ICU group compared to the ID group. Furthermore, contrary to non-COVID-19 patients, Proteobacteria were found to be abundant while Spirochaetes and Fusobacteria were depleted in COVID-19 patients.

Fecal microbiota transplantation in COVID-19 patients Liu et al. [34] were interested in the potential benefit of using faecal microbiota transplantation (FMT) on COVID-19 patients during their recovery. Eleven COVID-19 patients who had been discharged 1 month before the start of the study were recruited and received FMT for 4 days in the form of an oral capsule. Blood and stool samples were collected before and after FMT to observe any immunological profile alterations or changes in the composition of the gut microbiota. Upon receiving FMT, there was a decrease in the naive B cells and an increase in memory B cells and non-switched B cells, suggesting the treatment may have positive effects on the subjects' immune system. FMT also resulted in an improvement of dysbiosis post COVID-19 infection. At the same time, 5 out of 11 subjects reported GI symptoms after the recovery from COVID-19, and upon receiving FMT, all 5 patients reported a relief of their symptoms [34].

\section{Studies investigating the association between airway microbiota and COVID-19}

Among the 11 studies that investigated the association between airway microbiota and COVID-19, 8 studies concluded that there was an association between COVID-19 infection and alteration of airway microbiota composition $[37-40,42,43,45,46]$, while three studies suggested that there was no significant difference [34, 40, 45]. Six studies concluded that COVID-19 infection increased the coverage of opportunistic pathogens in the airway [37, 39, 40, 42,
$45,46]$, while 2 studies reported a reduction in commensal bacteria in the airway due to COVID-19 [35, 44] (Table 3).

Airway Microbiota in COVID-19 patients vs. patients infected with other viruses vs healthy participants Rueca et al. [37] examined the differences in the nasopharyngeal microbiota population among COVID-19 patients, other coronavirus patients and healthy individuals. They reported a decrease in nasopharyngeal microbiota richness among the COVID-19 patients, particularly in those admitted to the ICU. Opportunistic pathogens such as Pseudomonaceae presented exclusively in the COVID-19 ICU group [37].

Shen et al. [43] investigated the mutation rate of SARS$\mathrm{CoV}-2$ and also the variances in terms of nasopharyngeal microbiota composition between COVID-19 and community-acquired pneumonia (CAP) patients. The results of the study suggested that the mutation speed of SARS-CoV-2 allele may be comparable to that of the Ebola virus. On the other hand, comparison of airway microbiota of COVID-19 and CAP patients to that of healthy controls suggests lung dysbiosis, even though no significant changes were found in microbiome composition between COVID-19 and CAP patients [43].

Nardelli et al. [39] analysed the difference in the composition of the nasopharyngeal microbiota among COVID19 patients compared to recovered COVID-19 patients and healthy individuals. Compared to the healthy control group, it was found that the microbiome was significantly different in the COVID-19 group in comparison to the nonCOVID-19 group. This difference remained unchanged even after the patients' recovery. Interestingly, Fusobacterium periodonticum (FP), had a negative correlation with symptom severity. Previous studies have demonstrated the role of FP in the metabolism of sialic acid, which may be one of the key receptors of SARS-CoV-2 [48]. Therefore, the reduction of FP may increase patients' susceptibility to COVID-19 infection [39].

Rosas-Salazar et al. [38] examined if there were any differences in the composition of upper respiratory tract microbiota between mild COVID-19 patients and an uninfected control group. They reported a significant difference in the airway microbiome of nasal swab samples between the two groups. The difference was more prominent in COVID-19 patients with higher viral loads compared to those with lower viral loads [38].

Zhang et al. [42] examined possible changes in the diversity of the airway microbiome among COVID-19 patients compared to non-COVID pneumonia patients. They reported that the airway microbiome in samples from COVID19 patients was less diversified and more abundant with opportunistic pathogens. The research team also identified various differentially expressed host genes in samples from COVID-19 patients, most of which were related to cytokine 
signalling deregulation, suggesting an important role in the immunopathogenesis of COVID-19 [42].

Mostafa et al. [45] applied meta-genomic next-generation sequencing (mNGS) on COVID-19-positive and -negative nasopharyngeal swab specimens, and found that mNGS achieved $77.5 \%$ accuracy compared to regular diagnostic RT-PCR test. In addition, $12.5 \%$ of the samples showed a high abundance of opportunistic pathogens. COVID-19 samples also showed a significant decrease in their airway bacterial diversity which was directly proportionated to disease severity [45].

Merenstein et al. [46] reported a marked difference of airway microbiome communities between the COVID-19 patients and the healthy control group. Microbiota composition was significantly less diverse in patients presenting with severe disease. In addition to the above, the loss of microbiota diversity was also associated with a lower lymphocyte to neutrophil ratio, a well described biomarker linked to disease severity. However, the researchers acknowledged that it remains unclear whether the systemic immune response was directed by the airway microbiota or other factors [46].

Airway microbiota, the immune system and COVID-19 disease severity Ventero et al. [40] collected microbiota samples from nasopharyngeal swabs of COVID-19 patients and compared them to negative control subjects. The COVID-19 patients were further categorised according to disease severity. A significant difference in the nasopharynx microbiota composition between the two groups was reported. The network complexity of the microbiota was decreased in patients with more severe disease, suggesting a potential biomarker for COVID-19 severity [40].

Studies showing no association between COVID-19 infection and airway microbiota composition Contrary to the reports of other studies, Braun et al. [44] found no differences in the composition of the airway microbiome between samples from confirmed COVID-19 patients compared to negative COVID-19 patients [44]. Consistently, when De Maio et al. [36] compared the nasopharyngeal microbiota composition among COVID-19 and healthy controls they found that there were no significant differences in microbiota richness, diversity and composition between the SARSCoV-2-infected patients and the healthy control group [36].

Miao et al. [42] examined if there were any differences in the composition of the airway microbiota between critically ill COVID-19 patients compared to a non-COVID-19 control group. They reported that critically ill COVID-19 patients had a significantly higher co-infection rate $(81.3 \%)$ compared to those with severe $(5.1 \%)$ or mild disease $(0 \%)$. Antimicrobials were also commonly used in all patients $(100 \%$ of critically severe patients, $76.9 \%$ of severe patients and $21 \%$ of mild patients). In terms of differences in the airway microbiome, it was concluded that critically ill and intubated COVID-19 patients had a distinct airway microbiome compared to the non-intubated and non-COVID-19 patients. However, the microbiota profile in the intubated COVID-19 patients was similar to that of the intubated non-COVID-19 groups. Therefore, the research team suggested that the differences in airway microbiome in critically COVID-19 patients may be caused by intubation and mechanical ventilation, rather than COVID-19 infection per se [42].

\section{Studies investigating the association between both airway and gut microbiota and COVID-19}

Only one cohort study in China was identified in our search to have examined the association between both the gut and airway microbiota and COVID-19 [47] (Table 4). Xu et al. [47] conducted both throat and anal swabs on COVID-19 patients, non-COVID-19 patients and healthy adults to assess any changes between the airway and gut microbiota during the course of SARS-CoV-2 infection. The team reported that the diversity, richness and evenness of both the airway and the gut microbiome were significantly lower in COVID-19 patients, in comparison to both non-COVID-19 and the healthy control group. This alteration was gradually restored back to normal towards the end of their infection in both the upper respiratory tract and the intestine among COVID-19 patients with mild disease. Furthermore, the richness of the microbiome in both organ systems seemed to be negatively associated with the serum level of lipopolysaccharides. In terms of the microbiota composition, an elevated coverage of opportunistic pathogens was found in both the airway and the gut. Interestingly, the absence of functional bacteria, such as Bifidobacterium, Lactobacillus and Faecalibacterium, in the gut was found to be negatively correlated with the abundance of opportunistic pathogens [47].

\section{Discussion}

The aim of this review was to report the current evidence from research studies investigating the association between COVID-19 infection and the composition of the gut and airway microbiota. Nineteen studies concluded a correlation between airway and/or gut dysbiosis and SARS-CoV2 [26-35, 37-40, 42, 43, 45-47], while 3 studies failed to observe any significant association between the airway microbiome and SARS-CoV2 infection [36, 41, 44]. The findings of the current review suggest that the gut microbiota may have a more important role than the airway microbiota in COVID-19 infection. Specifically, the studies reported a higher colonization with opportunistic pathogens in both the gut and airway samples of SARS-CoV-2-infected patients. 
These opportunistic pathogens included Streptococcus, Clostridium, Enterococcus, Peptostreptococcaceae in the gut and Salmonella, Pseudomonadaceae, Bacteroidetes, Streptococcus, Staphylococcus Haemophilus, parainfluenzae, Neisseria, Rothia, Porphyromonas, Sarccharibacteria incertae sedis, Human influenza virus, Respiratory syncytial viruses and Human alphaherpesvirus 1 in the airway. In addition, there was a reported downregulation of commensal bacteria, such as butyrate-producing bacteria (BPB) Ruminococcaceae, Lachnospiraceae, Faecalibacterium and Bifidobacterium in the gut and Bacteroides and Bifidobacterium in the airway.

Interestingly, one of the studies addressed the potential linkage between the respiratory and the gut microbiome [47]. Compared to the gut, the respiratory tract is more susceptible to pathogenic invasions $[49,50]$, and thus dysbiosis, if any, in the respiratory tract is likely to occur earlier in comparison to intestinal dysbiosis [47]. Through microbeinduced inflammation and swallowing, bacteria may translocate from the oropharynx to the gastrointestinal tract, which potentially explains the association between respiratory and intestinal dysbiosis [50, 51].

Most of the studies included in the current review acknowledged that it may be difficult to identify whether the imbalance in the microbiota composition is the cause or consequence of SARS-CoV-2 infection. In fact, the samples from all studies were collected after the onset of SARSCoV-2 infection; therefore, there were no baseline microbiota compositions for researchers to compare microbiome diversity during and after SARS-CoV-2 infection. Animal studies may be able to provide some answers, as samples can be collected before, during and after the infection with SARS-CoV-2 to observe any changes in microbiota. By infecting macaques with the SARS-CoV virus, Sokol et al. [52] observed a clear cause and effect relationship between SARS-CoV-2 and intestinal microbiota composition. In particular, faecal samples from infected subjects were dominated by Bacteroidetes, Acinetobacter and Firmicutes. When comparing to SARS-CoV-2 viral load both nasopharyngeally and rectally, it was reported that the coverage of Acinetobacter was proportionated with the nasopharyngeal viral load, while the coverage of Peptostreptococcaceae was proportionated to the rectal viral load. These changes of microbiota ecology were also accompanied with a marked reduction of short-chain fatty acids (SCFAs) produced by the microbiome which may induce local immunomodulatory changes in the subjects [52]. The aforementioned immunomodulatory and taxonomic diversity changes in the microbiota composition, may account for the loss of microbiome functional redundancy and increased susceptibility to colonization from pathogenic bacteria in patients with COVID-19 infection.

Butyrate (one of the key SCFAs)-producing bacterium is significantly reduced during COVID-19 infection [27, 29,
$31,35,53]$. The resulting reduction of butyrate production in the gut may be linked to a pro-inflammatory state [54], thus increasing susceptibility to pulmonary viral infections, including COVID-19 [55-57]. Although evidence suggests that SCFA does not interfere with COVID-19 infection in the intestine [53], a reduction in SCFA may promote a systemic pro-inflammatory state in both macaques [52] and humans [58]. In support of this, dietary interventions may alter the composition of airway and intestinal microbiota, which may potentially affect the clinical course of COVID-19 infection by modulating systemic immune responses [59].

A decrease in SCFA can also downregulate the ACE2 [60], one of the key receptors of SARS-CoV-2 virus [61]. The depletion of ACE2 has been shown to promote epithelial damage in the intestine and thus increase susceptibility to inflammation in animal studies [62]. The abundance of SARS-CoV-2 virus in the gastrointestinal tract was proven to provide a gastrointestinal route for COVID-19 infection [63] which may worsen intestinal dysbiosis. Therefore, there is a pathogenic feedback loop between the reduced production of SCFA and SARS-CoV-2 [31]. A similar phenomenon can be observed in the airway through the downregulation of pharyngeal mucosal ACE2 by the imbalance of the airway microbiome [62, 64, 65].

The increase in opportunistic pathogens in the gut and in the airway during the course of COVID-19 also plays a significant role in worsening disease prognosis. Co-infection has been shown to be a key prognostic factor in COVID-19 [66]; for example, pulmonary aspergillosis was estimated to occur in 19-33\% of COVID-19 patients [67-69], and was reported in two of the studies included in this review [29, 31]; Pseudomonas is one of the most commonly reported pathogens isolated from COVID-19 patients with bacterial co-infection [70], which was reported in one study included in this review as well [37].

Several limitations have been identified in the studies included in the current review. All papers studied COVID-19 cases retrospectively and there was lack of longitudinal data to determine microbiota composition at baseline since this is not routinely assessed in the management of COVID-19 patients. More animal studies in the future may give us further insight on the progression of the microbiome alteration during the whole course of disease [71]. Another limitation is that 9 out of 10 gut microbiota studies came from either China or Hong Kong, while subjects from the airway microbiota studies were recruited in 6 different countries. This suggests that the literature on intestinal microbiome could contain a higher level of selection bias due to lack of geographic heterogeneity. Moreover, the high average median ages in our analysis may represent a relatively homogeneous demographic exposed to similar environmental factors. This could affect the objectivity when studying the patients' microbiota profiles [72]. The small average sample size of 
all studies is also an important limitation of the studies presented in the current paper. However, it is important to note that most studies were pilot studies due to the recent occurrence of COVID-19 pandemic. In addition, only one study included non-hospitalised COVID-19 patients [40], while 5 studies recruited patients from the Intensive Care Unit for comparison [27, 35, 37, 40, 41]. The composition of the microbiota could have been affected by the hospital [73] and ICU [74] environment, and may have therefore affected the objectivity of the studies. Furthermore, only 4 out of 22 studies excluded subjects who received antimicrobial and/ or probiotic treatment prior to the sample collection [26, 31, 34,38 ], while 9 studies chose to include these participants $[27,28,30,32,35,41,43,46,47]$ and 9 studies did not report this information [29, 33, 36, 37, 39, 40, 42, 44, 45]. In addition, with the exception of one study [35], the remaining studies did not report the time of sample collection in regards to the administration of antibiotic or probiotic. In fact, the use of antimicrobials have been shown to affect intestinal [75] and respiratory tract microbiota composition [49], therefore introducing bias in the findings. However, the difficulty to exclude these subjects is understandable, especially when antibiotics are commonly prescribed among critically ill COVID-19 patients [30]. In terms of sample collection, although certain studies suggest that rectal swabs are inferior to faecal sample collection [76], other studies suggested that they may be interchangeable [77, 78]. However, in airway microbiota studies, bronchoalveolar fluid lavage has been found to be a better sample collection method compared to nasopharyngeal swabs or throat swabs [79, 80], suggesting a better alternative for future study design. On the other hand, the composition of the microbiota may be easily affected during the process of sample collection by the personnel involved in sample collection as well as the hospital environment [81].

\section{Conclusion}

Our current review supports that there is overwhelming evidence that significant changes of the gut/airway microbiota composition are associated with SARS-CoV-2 infection and influence disease progression and prognosis. In particular, the gut microbiota may have a more important role than the airway microbiota in COVID-19 infection. However, studies with larger cohorts, longer duration, broader age group, more diverse subject demographics from different geographical locations, as well as studies that take into consideration provision of antimicrobials and probiotic administration in COVID-19 patients are needed to derive more conclusive results on identifying individualized changes of microbiota composition alterations of the gut-lung axis during SARS-CoV-2 infection. The completion of such studies will derive more conclusive evidence of the role of the microbiota in COVID-19 disease and will allow the development of better management strategies to be applied in combating COVID-19. As the pandemic progresses, a novel group of chronic patients with long-COVID is expected to pose a new challenge for healthcare settings worldwide. Sustained loss of intestinal microbiota diversity could be implicated in prolonged immunological changes that, in theory, could account for chronic symptoms associated with SARS-CoV-2 infection. Unravelling the dynamics of microbiome changes in the context of COVID-19 may offer a novel target for therapeutic interventions that extend beyond the acute phase of the disease.

Supplementary Information The online version contains supplementary material available at https://doi.org/10.1007/s15010-021-01715-5.

Acknowledgements Search terms were designed with the support of Mrs Carrie Rodomar, Head Librarian at the University of Nicosia Medical School.

Funding No external funding was received for this work

\section{Declarations}

Conflict of interest The authors declare that they have no conflict of interest.

\section{References}

1. Lipsitch M, Swerdlow DL, Finelli L. Defining the epidemiology of Covid-19: studies needed. N Engl J Med. 2020;382:1194-6. https://doi.org/10.1056/NEJMp2002125.

2. Chan JF-W, Yuan S, Kok K-H, To KK-W, Chu H, Yang J, et al. A familial cluster of pneumonia associated with the 2019 novel coronavirus indicating person-to-person transmission: a study of a family cluster. Lancet. 2020;395:514-23. https://doi.org/10.1016/ S0140-6736(20)30154-9.

3. Huang C, Wang Y, Li X, Ren L, Zhao J, Hu Y, et al. Clinical features of patients infected with 2019 novel coronavirus in Wuhan, China. Lancet. 2020;395:497-506. https://doi.org/10.1016/S01406736(20)30183-5.

4. Qiu H, Wu J, Hong L, Luo Y, Song Q, Chen D. Clinical and epidemiological features of 36 children with coronavirus disease 2019 (COVID-19) in Zhejiang, China: an observational cohort study. Lancet Infect Dis. 2020;20:689-96. https://doi.org/10.1016/ S1473-3099(20)30198-5.

5. Zhu J, Ji P, Pang J, Zhong Z, Li H, He C, et al. Clinical characteristics of 3062 COVID-19 patients: a meta-analysis. J Med Virol. 2020;92:1902-14. https://doi.org/10.1002/jmv.25884.

6. WHO. Coronavirus disease (COVID-2019) situation reports 2021

7. WHO. WHO Coronavirus (COVID-19) Dashboard 2021

8. van Doremalen N, Bushmaker T, Morris DH, Holbrook MG, Gamble A, Williamson BN, et al. Aerosol and surface stability of SARS-CoV-2 as compared with SARS-CoV-1. N Engl J Med. 2020:382:1564-7. https://doi.org/10.1056/NEJMc2004973.

9. Jia HP, Look DC, Shi L, Hickey M, Pewe L, Netland J, et al. ACE2 receptor expression and severe acute respiratory syndrome coronavirus infection depend on differentiation of human airway 
epithelia. J Virol. 2005;79:14614-21. https://doi.org/10.1128/JVI. 79.23.14614-14621.2005.

10. Wang W, Xu Y, Gao R, Lu R, Han K, Wu G, et al. Detection of SARS-CoV-2 in different types of clinical specimens. JAMA. 2020. https://doi.org/10.1001/jama.2020.3786.

11. Zhou P, Yang X-L, Wang X-G, Hu B, Zhang L, Zhang W, et al. A pneumonia outbreak associated with a new coronavirus of probable bat origin. Nature. 2020;579:270-3. https://doi.org/10.1038/ s41586-020-2012-7.

12. Mutua MP, Muya S, Muita GM. A general perspective of microbiota in human health and disease. Arch Clin Microbiol. 2020. https://doi.org/10.36648/1989-8436.11.2.106.

13. Vemuri R, Gundamaraju R, Shastri MD, Shukla SD, Kalpurath $\mathrm{K}$, Ball $\mathrm{M}$, et al. Gut microbial changes, interactions, and their implications on human lifecycle: an ageing perspective. BioMed Res Int. 2018;2018:1-13. https://doi.org/10.1155/2018/4178607.

14. Vemuri R, Shankar EM, Chieppa M, Eri R, Kavanagh K. Beyond just bacteria: functional biomes in the gut ecosystem including virome, mycobiome archaeome and helminths. Microorganisms. 2020;8:483. https://doi.org/10.3390/microorganisms8040483.

15. Yuki K, Fujiogi M, Koutsogiannaki S. COVID-19 pathophysiology: a review. Clin Immunol. 2020;215: 108427. https://doi.org/ 10.1016/j.clim.2020.108427.

16. Shi HY, Zhu X, Li WL, Mak JWY, Wong SH, Zhu ST, et al. Modulation of gut microbiota protects against viral respiratory tract infections: a systematic review of animal and clinical studies. Eur J Nutr. 2021. https://doi.org/10.1007/s00394-021-02519-x.

17. Chen C-J, Wu G-H, Kuo R-L, Shih S-R. Role of the intestinal microbiota in the immunomodulation of influenza virus infection. Microbes Infect. 2017;19:570-9. https://doi.org/10.1016/j.micinf. 2017.09.002.

18. Sencio V, Machado MG, Trottein F. The lung-gut axis during viral respiratory infections: the impact of gut dysbiosis on secondary disease outcomes. Mucosal Immunol. 2021;14:296-304. https:// doi.org/10.1038/s41385-020-00361-8.

19. Ichinohe T, Pang IK, Kumamoto Y, Peaper DR, Ho JH, Murray TS, et al. Microbiota regulates immune defense against respiratory tract influenza A virus infection. Proc Natl Acad Sci. 2011;108:5354-9. https://doi.org/10.1073/pnas.1019378108.

20. Chattopadhyay I, Shankar EM. SARS-CoV-2-indigenous microbiota nexus: Does gut microbiota contribute to inflammation and disease severity in COVID-19? Front Cell Infect Microbiol. 2021;11: 590874. https://doi.org/10.3389/fcimb.2021.590874.

21. Chhibber-Goel J, Gopinathan S, Sharma A. Interplay between severities of COVID-19 and the gut microbiome: implications of bacterial co-infections? Gut Pathog. 2021;13:14. https://doi.org/ 10.1186/s13099-021-00407-7.

22. Di Stadio A, Costantini C, Renga G, Pariano M, Ricci G, Romani L. The microbiota/host immune system interaction in the nose to protect from COVID-19. Life. 2020;10:345. https://doi.org/10. 3390/life10120345.

23. Peters MDJ, Godfrey CM, Khalil H, McInerney P, Parker D, Soares CB. Guidance for conducting systematic scoping reviews. Int J Evid Based Healthc. 2015;13:141-6. https://doi.org/10.1097/ XEB.0000000000000050.

24. Page MJ, McKenzie JE, Bossuyt PM, Boutron I, Hoffmann TC, Mulrow CD, et al. The PRISMA 2020 statement: an updated guideline for reporting systematic reviews. PLOS Med. 2021;18: e1003583. https://doi.org/10.1371/journal.pmed.1003583.

25. Page MJ, McKenzie JE, Bossuyt PM, Boutron I, Hoffmann TC, Mulrow CD, et al. The PRISMA 2020 statement: an updated guideline for reporting systematic reviews. BMJ. 2021;372:n71. https://doi.org/10.1136/bmj.n71.

26. Gu S, Chen Y, Wu Z, Chen Y, Gao H, Lv L, et al. Alterations of the gut microbiota in patients with Coronavirus Disease 2019 or
H1N1 influenza. Clin Infect Dis. 2020;71:2669-78. https://doi. org/10.1093/cid/ciaa709.

27. Tang L, Gu S, Gong Y, Li B, Lu H, Li Q, et al. Clinical significance of the correlation between changes in the major intestinal bacteria species and COVID-19 severity. Engineering. 2020;6:1178-84. https://doi.org/10.1016/j.eng.2020.05.013.

28. Zuo T, Liu Q, Zhang F, Lui GC-Y, Tso EY, Yeoh YK, et al. Depicting SARS-CoV-2 faecal viral activity in association with gut microbiota composition in patients with COVID-19. Gut. 2020. https://doi.org/10.1136/gutjnl-2020-322294.

29. Zuo T, Zhan H, Zhang F, Liu Q, Tso EYK, Lui GCY, et al. Alterations in fecal fungal microbiome of patients with COVID-19 during time of hospitalization until discharge. Gastroenterology. 2020;159:1302-1310.e5. https://doi.org/10.1053/j.gastro.2020.06. 048.

30. Yeoh YK, Zuo T, Lui GC-Y, Zhang F, Liu Q, Li AY, et al. Gut microbiota composition reflects disease severity and dysfunctional immune responses in patients with COVID-19. Gut. 2021;70:698706. https://doi.org/10.1136/gutjnl-2020-323020.

31. Lv L, Gu S, Jiang H, Yan R, Chen Y, Chen Y, et al. Gut mycobiota alterations in patients with COVID-19 and H1N1 infections and their associations with clinical features. Commun Biol. 2021;4:480. https://doi.org/10.1038/s42003-021-02036-x.

32. Yu L, Tong Y, Shen G, Fu A, Lai Y, Zhou X, et al. Immunodepletion with hypoxemia: a potential high risk subtype of Coronavirus Disease 2019. Infect Dis (Except HIV/AIDS). 2020. https://doi. org/10.1101/2020.03.03.20030650.

33. Chen Y, Gu S, Chen Y, Lu H, Shi D, Guo J, et al. Six-month follow-up of gut microbiota richness in patients with COVID-19. Gut. 2021. https://doi.org/10.1136/gutjnl-2021-324090.

34. Liu F, Ye S, Zhu X, He X, Wang S, Li Y, et al. Gastrointestinal disturbance and effect of fecal microbiota transplantation in discharged COVID-19 patients. J Med Case Rep. 2021;15:60. https:// doi.org/10.1186/s13256-020-02583-7.

35. Mazzarelli A, Giancola ML, Farina A, Marchioni L, Rueca M, Gruber CEM, et al. 16S rRNA gene sequencing of rectal swab in patients affected by COVID-19. PLoS ONE. 2021;16: e0247041. https://doi.org/10.1371/journal.pone.0247041.

36. De Maio F, Posteraro B, Ponziani FR, Cattani P, Gasbarrini A, Sanguinetti M. Nasopharyngeal microbiota profiling of SARSCoV-2 infected patients. Biol Proced Online. 2020;22:18. https:// doi.org/10.1186/s12575-020-00131-7.

37. Rueca M, Fontana A, Bartolini B, Piselli P, Mazzarelli A, Copetti $\mathrm{M}$, et al. Investigation of nasal/oropharyngeal microbial community of COVID-19 patients by $16 \mathrm{~S}$ rDNA sequencing. Int J Environ Res Public Health. 2021;18:2174. https://doi.org/10.3390/ ijerph18042174.

38. Rosas-Salazar C, Kimura KS, Shilts MH, Strickland BA, Freeman $\mathrm{MH}$, Wessinger BC, et al. SARS-CoV-2 infection and viral load are associated with the upper respiratory tract microbiome. J Allergy Clin Immunol. 2021;147:1226-1233.e2. https://doi.org/ 10.1016/j.jaci.2021.02.001.

39. Nardelli C, Gentile I, Setaro M, Di Domenico C, Pinchera B, Buonomo AR, et al. Nasopharyngeal microbiome signature in COVID-19 positive patients: Can we definitively get a role to fusobacterium periodonticum? Front Cell Infect Microbiol. 2021;11: 625581. https://doi.org/10.3389/fcimb.2021.625581.

40. Ventero MP, Cuadrat RRC, Vidal I, Andrade BGN, Molina-Pardines C, Haro-Moreno JM, et al. Nasopharyngeal microbial communities of patients infected with SARS-CoV-2 that developed COVID-19. Front Microbiol. 2021;12: 637430. https://doi.org/ 10.3389/fmicb.2021.637430.

41. Miao Q, Ma Y, Ling Y, Jin W, Su Y, Wang Q, et al. Evaluation of superinfection, antimicrobial usage, and airway microbiome with metagenomic sequencing in COVID-19 patients: a cohort study 
in Shanghai. J Microbiol Immunol Infect. 2021. https://doi.org/ 10.1016/j.jmii.2021.03.015.

42. Zhang H, Ai J-W, Yang W, Zhou X, He F, Xie S, et al. Metatranscriptomic Characterization of COVID-19 Identified A Host Transcriptional Classifier Associated With Immune Signaling. Clin Infect Dis. 2021;73(3):376-385. https://doi.org/10.1093/cid/ciaa6 63.

43. Shen Z, Xiao Y, Kang L, Ma W, Shi L, Zhang L, et al. Genomic diversity of severe acute respiratory Syndrome-Coronavirus 2 in patients with Coronavirus Disease 2019. Clin Infect Dis. 2020;71:713-20. https://doi.org/10.1093/cid/ciaa203.

44. Braun T, Halevi S, Hadar R, Efroni G, Glick Saar E, Keller N, et al. SARS-CoV-2 does not have a strong effect on the nasopharyngeal microbial composition. Sci Rep. 2021;11:8922. https://doi.org/10.1038/s41598-021-88536-6.

45. Mostafa HH, Fissel JA, Fanelli B, Bergman Y, Gniazdowski V, Dadlani M, et al. Metagenomic next-generation sequencing of nasopharyngeal specimens collected from confirmed and suspect COVID-19 patients. MBio. 2020;11:e01969-e2020. https://doi. org/10.1128/mBio.01969-20.

46. Merenstein C, Liang G, Whiteside SA, Cobián-Güemes AG, Merlino MS, Taylor LJ, et al. Signatures of COVID-19 severity and immune response in the respiratory tract microbiome. Infect Dis (Except HIV/AIDS). 2021. https://doi.org/10.1101/2021.04.02. 21254514.

47. Xu R, Lu R, Zhang T, Wu Q, Cai W, Han X, et al. Temporal association between human upper respiratory and gut bacterial microbiomes during the course of COVID-19 in adults. Commun Biol. 2021;4:240. https://doi.org/10.1038/s42003-021-01796-w.

48. Morniroli D, Giannì ML, Consales A, Pietrasanta C, Mosca F. Human sialome and Coronavirus Disease-2019 (COVID19) pandemic: An understated correlation? Front Immunol. 2020;11:1480. https://doi.org/10.3389/fimmu.2020.01480.

49. Man WH, de Steenhuijsen Piters WAA, Bogaert D. The microbiota of the respiratory tract: gatekeeper to respiratory health. Nat Rev Microbiol. 2017;15:259-70. https://doi.org/10.1038/nrmicro. 2017.14.

50. Dang AT, Marsland BJ. Microbes, metabolites, and the gut-lung axis. Mucosal Immunol. 2019;12:843-50. https://doi.org/10.1038/ s41385-019-0160-6.

51. Wypych TP, Wickramasinghe LC, Marsland BJ. The influence of the microbiome on respiratory health. Nat Immunol. 2019;20:1279-90. https://doi.org/10.1038/s41590-019-0451-9.

52. Sokol H, Contreras V, Maisonnasse P, Desmons A, Delache B, Sencio $\mathrm{V}$, et al. SARS-CoV-2 infection in nonhuman primates alters the composition and functional activity of the gut microbiota. Gut Microbes. 2021;13:1-19. https://doi.org/10.1080/19490 976.2021.1893113.

53. Pascoal LB, Rodrigues PB, Genaro LM, dos Gomes ABSP, Toledo-Teixeira DA, Parise PL, et al. Microbiota-derived shortchain fatty acids do not interfere with SARS-CoV-2 infection of human colonic samples. Gut Microbes. 2021;13:1-9. https://doi. org/10.1080/19490976.2021.1874740.

54. Qin N, Zheng B, Yao J, Guo L, Zuo J, Wu L, et al. Influence of H7N9 virus infection and associated treatment on human gut microbiota. Sci Rep. 2015;5:14771. https://doi.org/10.1038/srep1 4771.

55. Friedland RP, Haribabu B. The role for the metagenome in the pathogenesis of COVID-19. EBioMedicine. 2020;61: 103019. https://doi.org/10.1016/j.ebiom.2020.103019.

56. Tang Y, Liu J, Zhang D, Xu Z, Ji J, Wen C. Cytokine storm in COVID-19: the current evidence and treatment strategies. Front Immunol. 2020;11:1708. https://doi.org/10.3389/fimmu.2020. 01708.

57. Chemudupati M, Kenney AD, Smith AC, Fillinger RJ, Zhang $\mathrm{L}$, Zani A, et al. Butyrate reprograms expression of specific interferon-stimulated genes. J Virol. 2020;94:e00326-e420. https://doi.org/10.1128/JVI.00326-20.

58. Esquivel-Elizondo S, Ilhan ZE, Garcia-Peña EI, KrajmalnikBrown R. Insights into butyrate production in a controlled fermentation system via gene predictions. mSystems. 2017. https:// doi.org/10.1128/mSystems.00051-17.

59. Gasmi A, Tippairote T, Mujawdiya PK, Peana M, Menzel A, Dadar M, et al. The microbiota-mediated dietary and nutritional interventions for COVID-19. Clin Immunol. 2021;226: 108725. https://doi.org/10.1016/j.clim.2021.108725.

60. Robinson CM, Pfeiffer JK. Viruses and the microbiota. Annu Rev Virol. 2014;1:55-69. https://doi.org/10.1146/annurev-virol ogy-031413-085550.

61. Melin AD, Janiak MC, Marrone F, Arora PS, Higham JP. Comparative ACE2 variation and primate COVID-19 risk. Commun Biol. 2020;3:641. https://doi.org/10.1038/s42003-020-01370-w.

62. Hashimoto T, Perlot T, Rehman A, Trichereau J, Ishiguro H, Paolino M, et al. ACE2 links amino acid malnutrition to microbial ecology and intestinal inflammation. Nature. 2012;487:477-81. https://doi.org/10.1038/nature11228.

63. Xiao F, Tang M, Zheng X, Liu Y, Li X, Shan H. Evidence for gastrointestinal infection of SARS-CoV-2. Gastroenterology. 2020;158:1831-1833.e3. https://doi.org/10.1053/j.gastro.2020. 02.055 .

64. Koester ST, Li N, Lachance DM, Morella NM, Dey N. Variability in digestive and respiratory tract Ace 2 expression is associated with the microbiome. PLoS ONE. 2021;16: e0248730. https:// doi.org/10.1371/journal.pone.0248730.

65. Khatiwada S, Subedi A. Lung microbiome and coronavirus disease 2019 (COVID-19): possible link and implications. Hum Microbiome J. 2020;17: 100073. https://doi.org/10.1016/j.humic. 2020.100073.

66. Cox MJ, Loman N, Bogaert D, O'Grady J. Co-infections: potentially lethal and unexplored in COVID-19. Lancet Microbe. 2020;1: e11. https://doi.org/10.1016/S2666-5247(20)30009-4.

67. Alanio A, Dellière $S$, Fodil S, Bretagne $S$, Mégarbane B. Prevalence of putative invasive pulmonary aspergillosis in critically ill patients with COVID-19. Lancet Respir Med. 2020;8:e48-9. https://doi.org/10.1016/S2213-2600(20)30237-X.

68. Koehler P, Cornely OA, Böttiger BW, Dusse F, Eichenauer DA, Fuchs F, et al. COVID-19 associated pulmonary aspergillosis. Mycoses. 2020;63:528-34. https://doi.org/10.1111/myc.13096.

69. van Arkel A, Rijpstra TA, Belderbos H, van Wijngaarden P, Verweij PE, Bentvelsen RG. COVID-19-associated pulmonary aspergillosis. Am J Resp Crit Care Med. 2020;202(1):132-135. https:// doi.org/10.1164/rccm.202004-10.

70. Qu J, Cai Z, Liu Y, Duan X, Han S, Liu J, et al. Persistent bacterial coinfection of a COVID-19 Patient caused by a genetically adapted pseudomonas aeruginosa chronic colonizer. Front Cell Infect Microbiol. 2021;11: 641920. https://doi.org/10.3389/fcimb. 2021.641920.

71. Yang T, Chakraborty S, Saha P, Mell B, Cheng X, Yeo J-Y, et al. Gnotobiotic rats reveal that gut microbiota regulates colonic mRNA of Ace2, the receptor for SARS-CoV-2 infectivity. Hypertension. 2020. https://doi.org/10.1161/HYPERTENSIONAHA. 120.15360 .

72. Scepanovic P, Hodel F, Mondot S, Partula V, Byrd A, The Milieu Intérieur Consortium, et al. A comprehensive assessment of demographic, environmental, and host genetic associations with gut microbiome diversity in healthy individuals. Microbiome. 2019;7:130. https://doi.org/10.1186/s40168-019-0747-x.

73. Ticinesi A, Milani C, Lauretani F, Nouvenne A, Mancabelli L, Lugli GA, et al. Gut microbiota composition is associated with polypharmacy in elderly hospitalized patients. Sci Rep. 2017;7:11102. https://doi.org/10.1038/s41598-017-10734-y. 
74. Aardema H. Marked changes in gut microbiota in cardio-surgical intensive care patients: a longitudinal cohort study. Front Cell Infect Microbiol. 2020;9:10.

75. Ramirez J, Guarner F, Bustos Fernandez L, Maruy A, Sdepanian VL, Cohen H. Antibiotics as major disruptors of gut microbiota. Front Cell Infect Microbiol. 2020;10: 572912. https://doi.org/10. 3389/fcimb.2020.572912.

76. Mottawea W, Butcher J, Li J, Abujamel T, Manoogian J, Mack D, et al. The mucosal-luminal interface: an ideal sample to study the mucosa-associated microbiota and the intestinal microbial biogeography. Pediatr Res. 2019;85:895-903. https://doi.org/10.1038/ s41390-019-0326-7.

77. Bassis CM, Moore NM, Lolans K, Seekatz AM, Weinstein RA, For the CDC Prevention Epicenters Program, et al. Comparison of stool versus rectal swab samples and storage conditions on bacterial community profiles. BMC Microbiol. 2017;17:78. https://doi. org/10.1186/s12866-017-0983-9.

78. Reyman M, van Houten MA, Arp K, Sanders EAM, Bogaert D. Rectal swabs are a reliable proxy for faecal samples in infant gut microbiota research based on 16S-rRNA sequencing. Sci Rep. 2019;9:16072. https://doi.org/10.1038/s41598-019-52549-z.

79. Marsh RL, Kaestli M, Chang AB, Binks MJ, Pope CE, Hoffman $\mathrm{LR}$, et al. The microbiota in bronchoalveolar lavage from young children with chronic lung disease includes taxa present in both the oropharynx and nasopharynx. Microbiome. 2016;4:37. https:// doi.org/10.1186/s40168-016-0182-1.

80. Van Driessche L, Valgaeren BR, Gille L, Boyen F, Ducatelle R, Haesebrouck F, et al. A deep nasopharyngeal swab versus nonendoscopic bronchoalveolar lavage for isolation of bacterial pathogens from preweaned calves with respiratory disease. J Vet Intern Med. 2017;31:946-53. https://doi.org/10.1111/jvim.14668.

81. Kim D, Hofstaedter CE, Zhao C, Mattei L, Tanes C, Clarke E, et al. Optimizing methods and dodging pitfalls in microbiome research. Microbiome. 2017;5:52. https://doi.org/10.1186/ s40168-017-0267-5. 